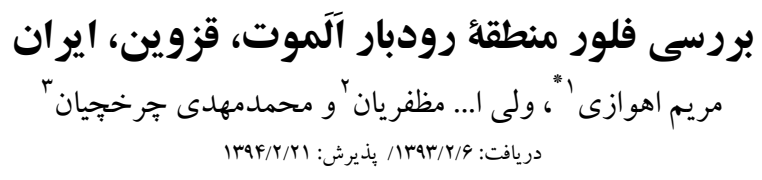

$$
\begin{aligned}
& \text { 'مركز تحقيقات گياهان دارويى، يُزوهشكدة گياهان دارويى جهاد دانشكاهى، كرج }
\end{aligned}
$$

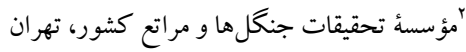

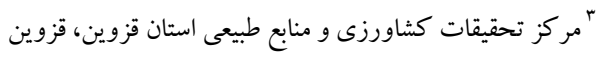

Maryame_ahvazi@yahoo.com:مسئول مكاتبات"

$$
\begin{aligned}
& \text { جكيده. تحقيق حاضر فلور منطقة رودبار آلموت در شمالشرق استان قزوين، واقع در دامنهاى جنوبى البرز مركزى را مطالعه مى كند. آلموت به دو بخش آلموت بالاو }
\end{aligned}
$$

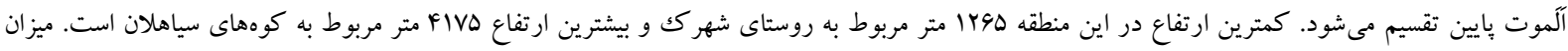

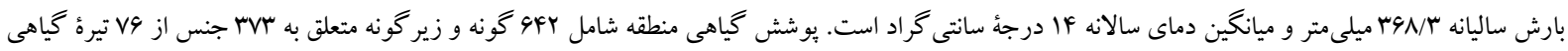

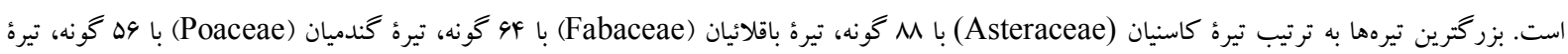

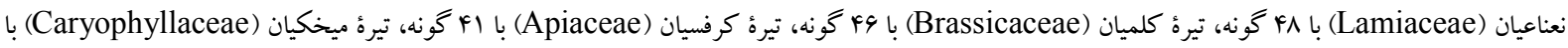

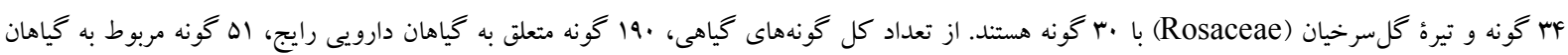

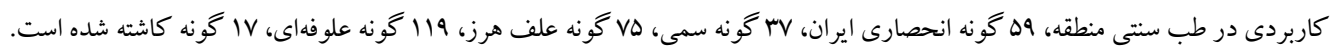

$$
\begin{aligned}
& \text { وازههاى كليدى. عناصر رويشى، گياهان دارويى، گياهان سمى، فلورستيك }
\end{aligned}
$$

\title{
The investigation of flora in Roodbar Alamut region, Ghazvin, Iran
}

\author{
Maryam Ahvazi ${ }^{*}$, Valiollah Mozaffarian ${ }^{2}$ and Mohammad Mehdi Charkhchian ${ }^{3}$ \\ Received 28.08.2014/ Accepted 11.05.2015
}

\footnotetext{
${ }^{1}$ Medicinal Plants Research Center, Institute of Medicinal Plants, ACECR, Karaj, Iran

${ }^{2}$ Research Institute of Forests and Rangeland, Tehran, Iran

${ }^{3}$ Research Institute of Forests and Rangeland, Ghazvin, Iran

*Correspondent author: Maryame_ahvazi@yahoo.com
}

\begin{abstract}
This paper explores flora of Rudbar Alamut region in NE of Ghazvin province situated in the south slope of central Alborz Chain Mountains. Alamut is divided into two parts, upper Alamut and lower Alamut. The minimum height in the region is $1265 \mathrm{~m}$, located in Shahrak village, while the maximum height is $4175 \mathrm{~m}$, located in Siahlan Mountain. Average of annual precipitation is $368.3 \mathrm{~mm}$ and annual average temperature is $14^{\circ} \mathrm{C}$. This reveals that 642 species and subspecies belong to 373 genera and 76 plant families growing in the region. The largest families are Asteraceae with 88 species, Paoilionaceae with 64, Poaceae with 56, Lamiaceae with 48, Brassicaceae with 46, Apiaceae with 41, Charyophyllaceae with 34 and Rosaceae with 30, respectively. Nearly 190 species are medicinal plants, of which 51 are used traditionally by local inhabitatants, 59 species are endemic, 37 species are poisonous for animal and rarely for man, 75 species are weeds, 119 species are forage, and 17 species are cultivated.
\end{abstract}

Keywords. chorotype, medicinal plants, poisonous plants, floristics 
در لغتنامة فارسى آمده است كه آلموت از دو كلمة آله و آموت تشكيل شده است و به معناى آشيانه عقاب است (آمير،

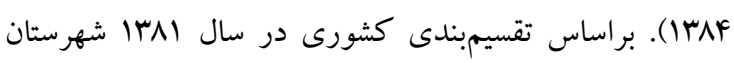
قزوين داراى شش بخش مركزى، رودبار آلموت، رودبار

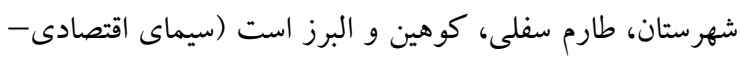

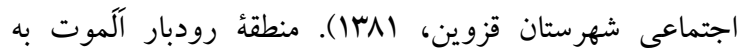

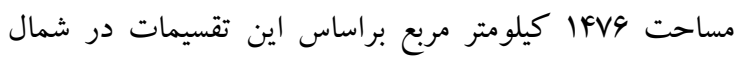

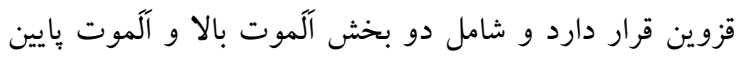
است (طرح توسعه و عمران حوضة آبريز شاهرود، المبار).

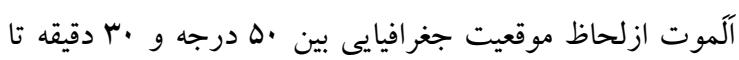

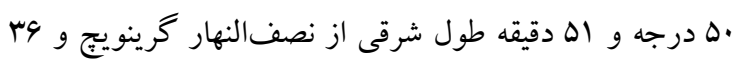

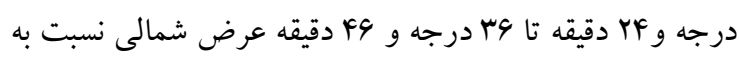

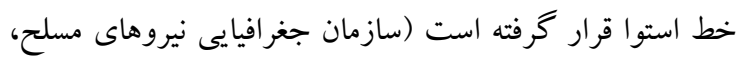
IrVa

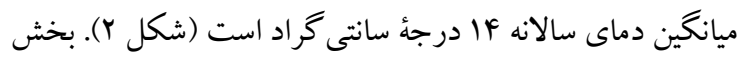

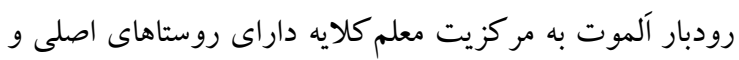
برجمعيتى نظير شمس كلايه، كازرُرخان، باغكلايه، سائين كلايه،

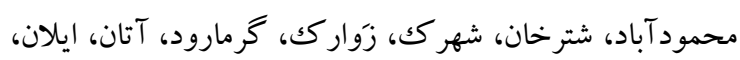

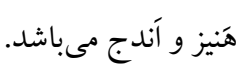

كياهناسى يكى از علوم قديمى است كه سابقهُ آن به دوران

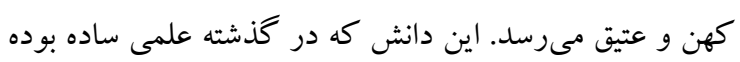
و در مواردى فقط به شناخت معدودى از گياهان و نحوهُ استفاده

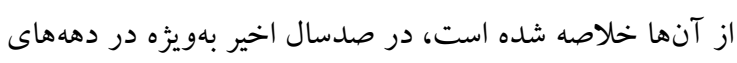

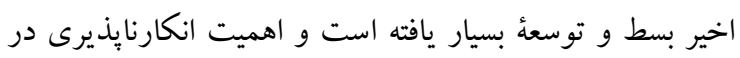
كشاورزى و رشتههاى مختلف آن، داروسازى و درمان، شناخت و توان محيط زيست، بومشناسى و سرانجام در بهره گيرى بيشتر

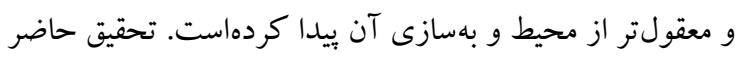

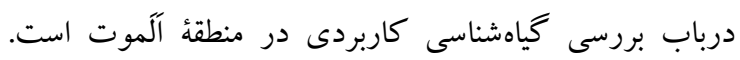

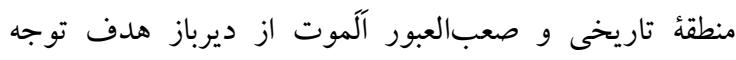

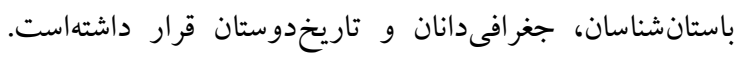

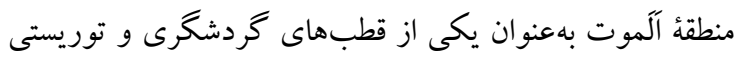

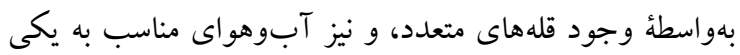

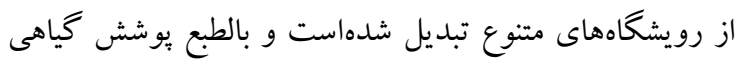

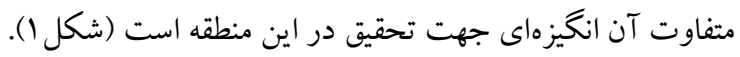

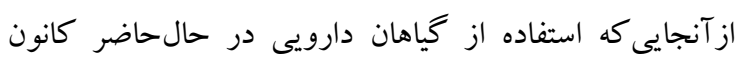

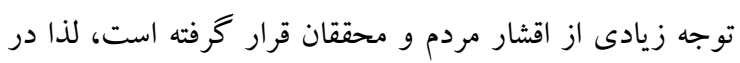

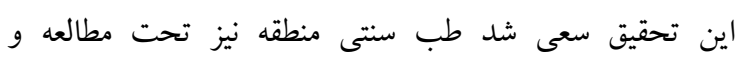

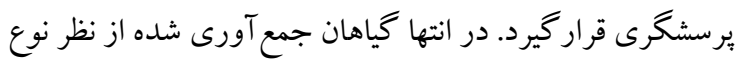

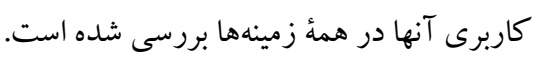

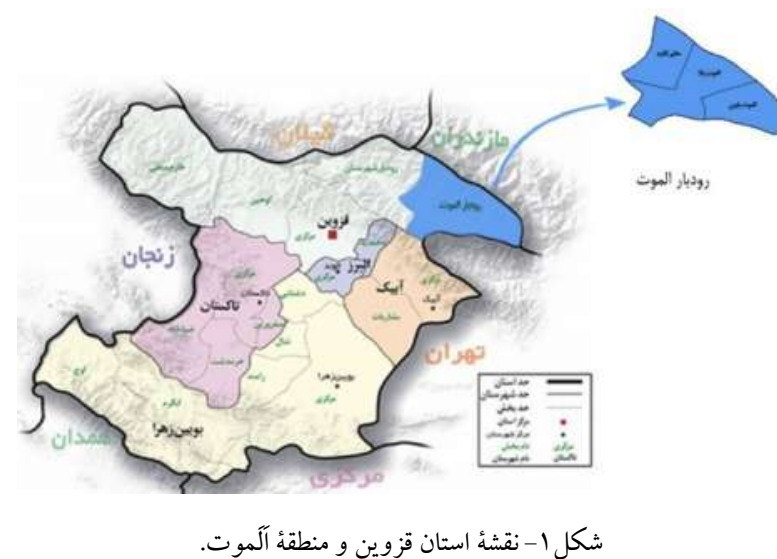

Fig. 1. Map of Ghazvin province and Alamut region. 
كارشناسان ادارهٔ كل هواشناسى استان قزوين، شمال شرق استان داراى آبوهواى مرطوب فراسرد و با افزايش دما، اقليم خيلى مرطوب فراسرد نوع ا تخمين زده شده است. در اقليم يايينتر آب و هواى منطقه مديترانهاى سرد است.
رشتهٔ اصلى كوه البرز با قله هاى مشهور تخت سليمان و سياهلان، زرد سياه، اَجرا، خَشجال حد فاصل بين ألَموت و درياى خزر واقع شده است. ازجمله قلههاى مرتفع و مهم منطقة آلموت مى توان به موارد زير اشاره كرد:

سياهلان با ارتفاع FIVD متر در آلموت بالا، خَشَحال با ارتفاع

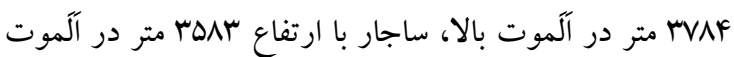

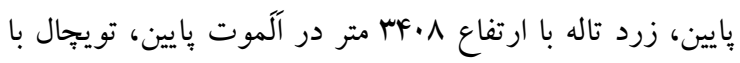

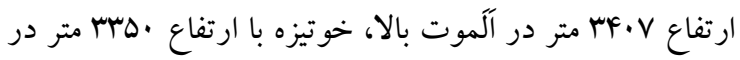

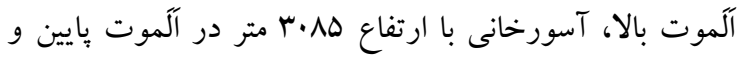

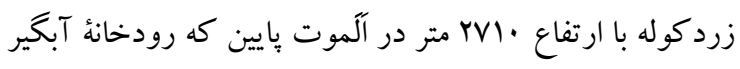

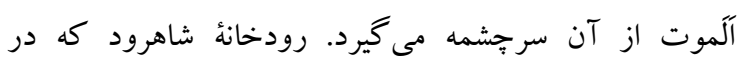
كوهستان البرز جريان دارد از مهمترين رودهاى اين منطقه بهشمار مى آيد (جعفرى،

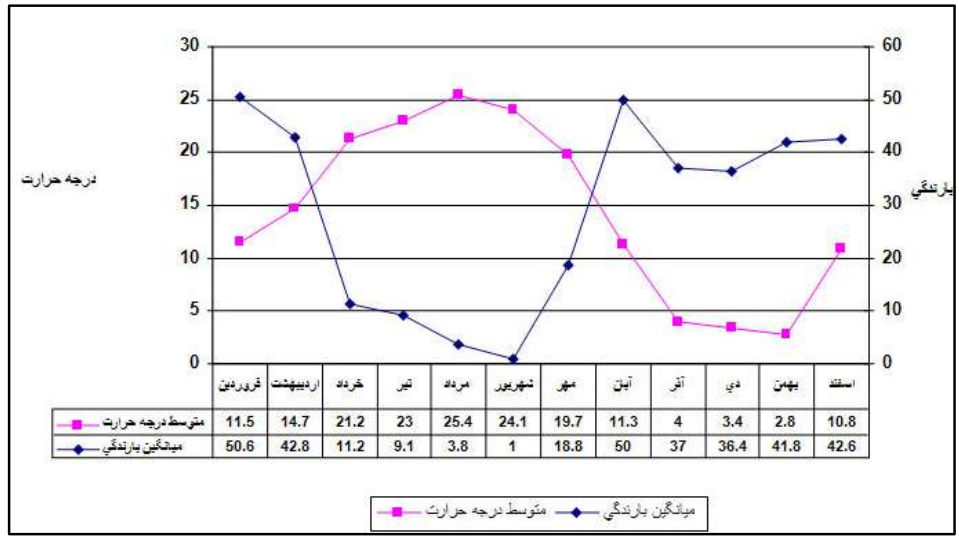

شكل r - منحنى آمبروترميك ايستاه هواشناسى معلم كلايه.

Fig. 2. Amberothermic curve of moallem-kolayeh station.

كونهاى بسيار از نظر مطالعات گياهشناسى هدف توجه قرار روش تحقيق

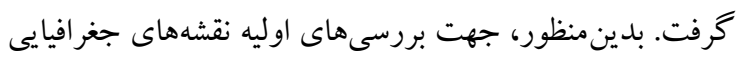

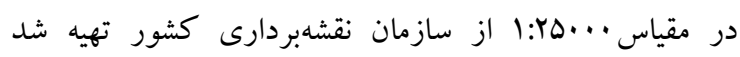
(سازمان نقشهبردارى كل كشور، IrNF). ستبس وضعيت راههاى ارتباطى، يوشش گياهى و مناطق مسكونى مورد مطالعه قرار گرفت. باتوجه به فصل رويش گياهان يككساله، دوساله و جند منطقهُ تحت مطالعه با وسعت نسبتاً زياد و اختلاف ارتفاعى

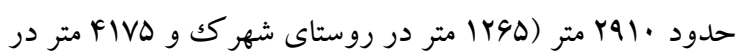
كوههاى سياهلان) در شيبهاى جنوبى ارتفاعات البرز مر كزى و منطقة آلموت رود كه در حوضدُ شاهرود واقع شده و با تنوع 
بس از بررسى يوشش گياهى اين منطقه فهرست كاملى از گياهان

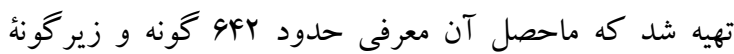

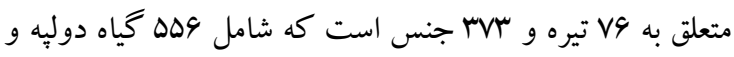
AV

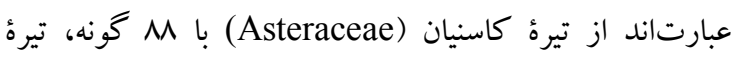
باقلائيان (Fabaceae) با عو كونه، تيرة نعناعيان (Lamiaceae)

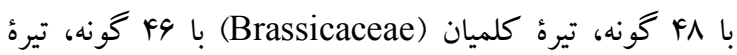

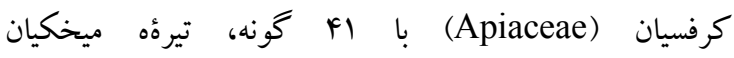
با با (Caryophyllaceae)

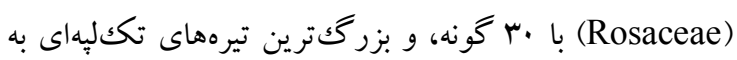

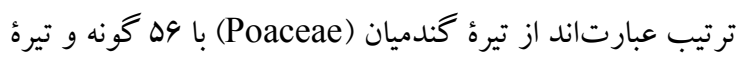

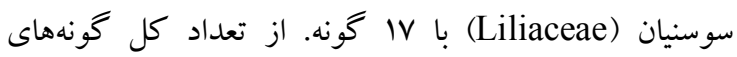

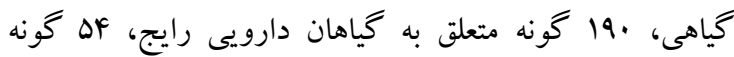

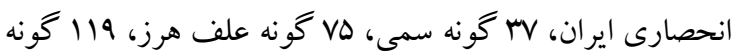

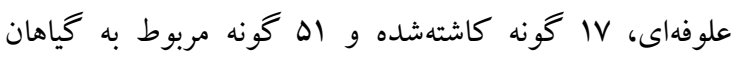
كاربردى در طب سنتى منطقه است.

طيف اشكال زيستى گياهى غالب منطقه، همى كريتوفيت با بهه

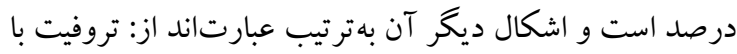

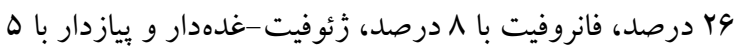
درصد، كامفيت با F درصد، زئوفيت- ساقه زيرزمينى با ب درصد

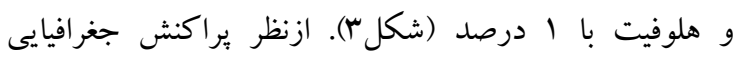
كياهان منطقه اهله درصد ايرانو-تورانى، الس درصد ايرانو - درصدي

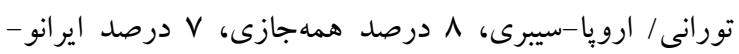

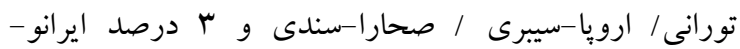
تورانى/صحارا-سندى هستند (شكل f).
ساله و با توجه به اينكه جهت شناسايى آنها نياز به كياه كامل

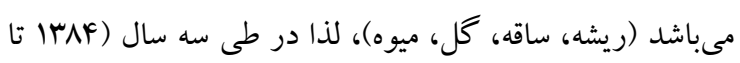

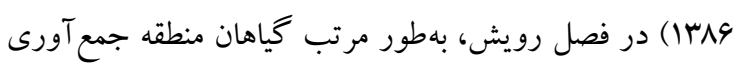

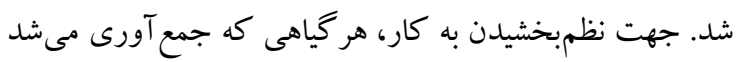
با يكك كد مشخص و دركيسٔ مخصوص جمع آورى كذاشته

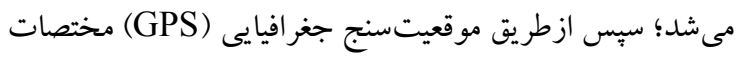

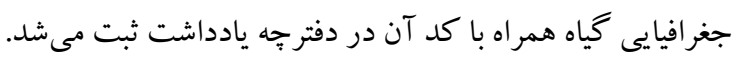

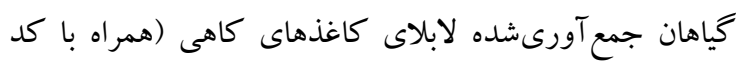

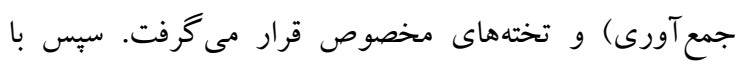

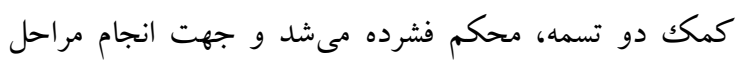

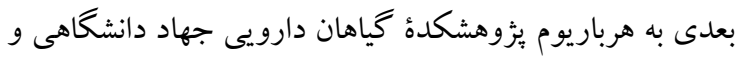
مؤسسؤ تحقيقات جنكل ها و مراتع تهران منتقل مى شيد. در شناسايى جنسها و گونههاى گياهى مطابق با روش

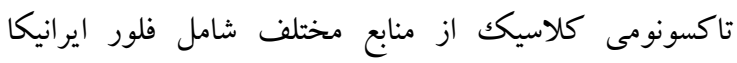

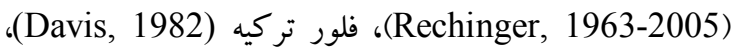
فلور عراق (Townsend 1966-1985)، فلور استان خوزستان

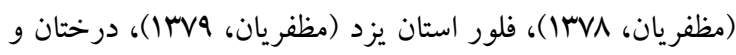
درختجههاى ايران (مظفريان،

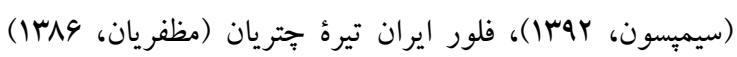
استفاده شد. اشكال زيستى گياهان براساس سيستم رونكيه تعيين

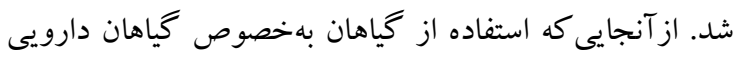
در حالحاضر بسيار مورد توجه اقشار مردم و محققان قرار كرفتهاست، در اين تحقيق سعى شد طب سنتى منطقه نيز تحت

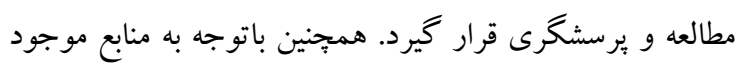

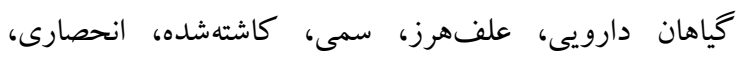
علوفهاى و برخى كاربردهاى معمول آنها بررسى شد علد (ميرحيدر،

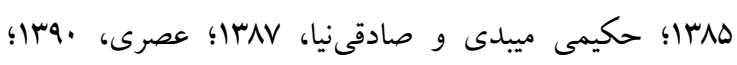

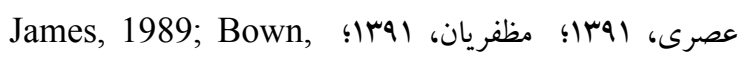
1995; Fleming, 1998; Ghahremaninejad et al., .2012) 


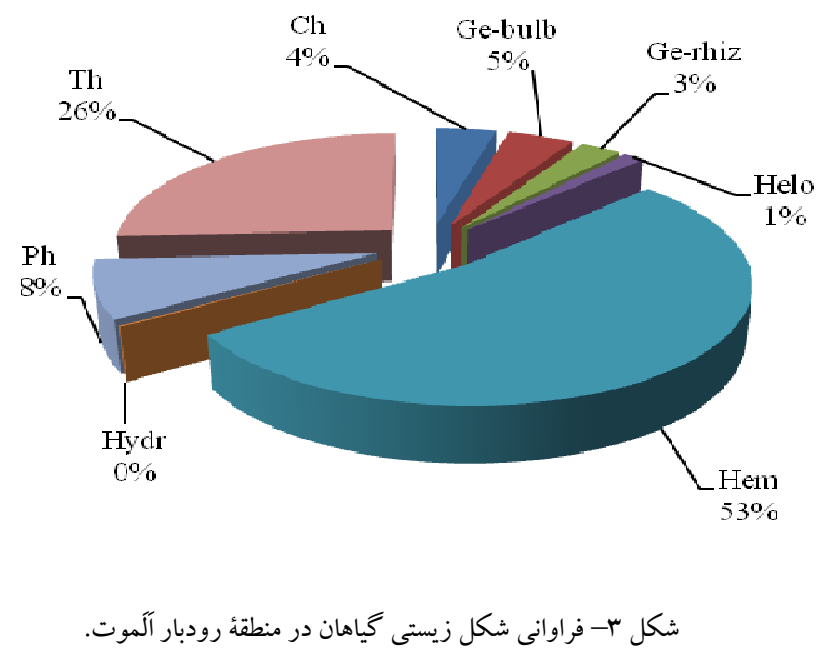

Fig. 3. Life form spectrum of plants in Roodbar Alamut region.

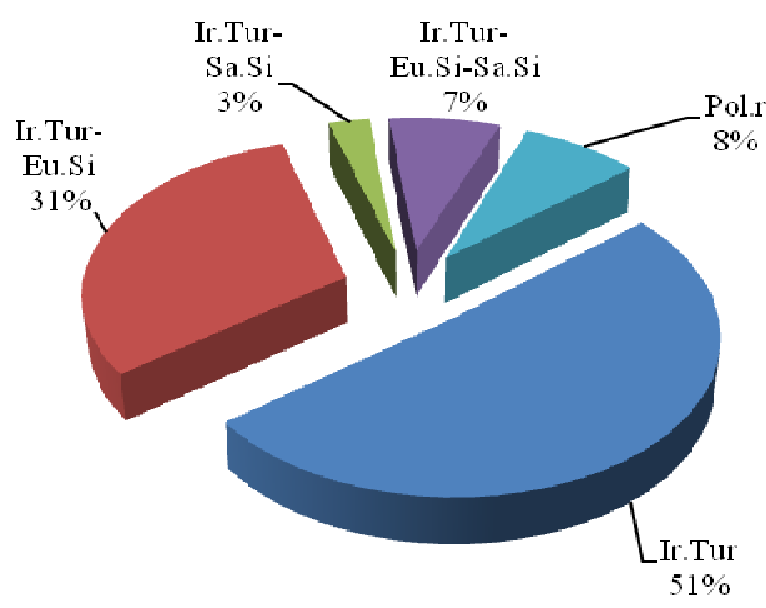

شكل F- درصد فراوانى انتشار جغر افيايى گياهان منطقه.

Fig. 4. Percentage of main chorotypes of plants studied in Roodbar Alamut region.

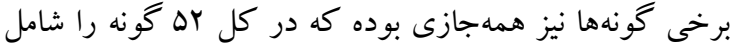
مىشوند. عناصر ايرانو -تورانى شامل و كr گُنه و عناصرى كه بهور مشترك ايرانو-تورانى/ ارويا - سيبرى هستند $19 V$ كونه مىباشد كه نشاندهندهُ براكنش نسبتاً خوب اين كونهها است. عناصرى كه در هر سه ناحيه ايرانو-تورانى و ارويا- سيبرى و و

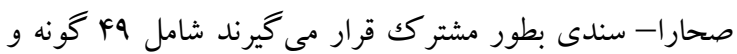
11 كونه نيز در نواحى ايرانو- تورانى/ صحارا- سندى قرار مى گيرند. رشته كوه البرز بهدليل داشتن بافت متنوعى از جينهبندى ضخيم و متناوب شامل سنگ آهك،، سنگك رس، سنگك ماسه و خاكستر آتشفشانى است. ازاينرو بوشش كياهى متنوعى نيز در اين

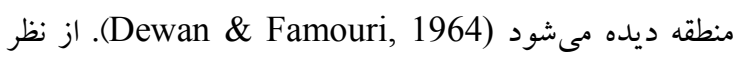
تقسيمبندى جغرافيايى گياهى اين منطقه جزو ناحئ ايرانو -

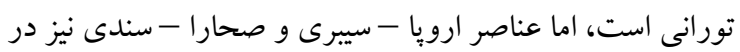
آن ديده مى شود. 
منطقه در جاى خود بهصورت گسترده بحث خو اهد شد، بِ در اينجا فقط جهت آشنايى به تعدادى از آنها اشاره شده است.

در يوشش گياهى اين منطقه گونهاى مرتعى مهمى كه ارزش اقتصادى و محافظتى زيادى دارند بهفراوانى يافت مى شوند كه

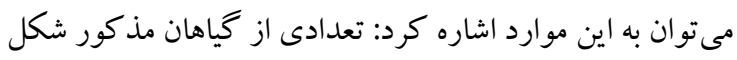
بشتهاى دارند و به دليل داشتن سيستم ريشهاى عميق و قوى از از

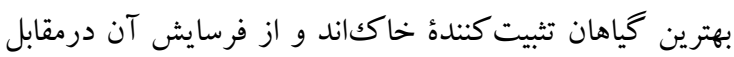
سيلابها جلو گيرى مى كنند (حكيمى ميبدى و صادقىنيا، لrNV ،(Hippomarathrum microcarpum) كافورى(Camphorosma) كوَن (Astragalus aegobrromus) (Pennisetum و ريش يرى (Acantholimon erinaceum) (Trifolium كخاهانى نظير مريم نخودى orientale) (Trifolium fragiferum)، شبدر توتفرنغئ)، fragiferum) شبدر سفيد (Trifolium repens) و آويشن كركآلود به دليل داشتن گل هاى فراوان و معطر (Thymus pubescens) موجب جذب زنبور عسل مىشود و ازاينرو در صنعت زنبور -

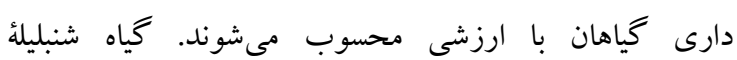

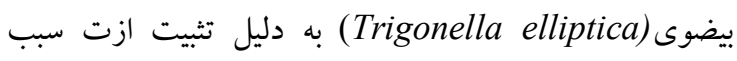
تقويت خاكك و رشد و توسعة گياهان ديخر مىشود و كياه ترشك البرزى (Rumex elbuesensis) داراى ريشههاى دارويى است و ريشههاى آن آهن جذب مى كند (جورى و

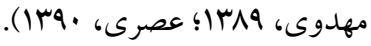

تعدادى از گونهاى انحصارى در اين منطقه نيز به جشم مىخورد كه برخى از آنها گونهاى نادر منطقه را شامل مىشود. از گونهاى نادر در منطقه مىتوان به گونهُ خللر اشاره كرد كه در اين تحقيق براى (Lathyrus alamutensis) (Mozafarian, اولينبار از منطقة آلموت جمع آورى شدائ

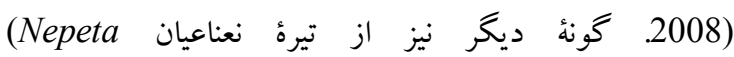
است كه اولينبار در اين منطقه شناسايى شده است (Jamzad, 1984).
از آنجايىكه طيف بيولوزيكى هر منطقه مىتواند اطلاعات مفيدى از وضع رويش و جامعه رويشى در اختيار ما بخذارد لذا هـا

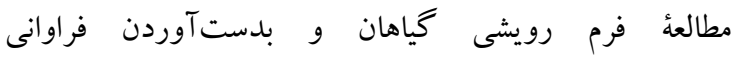
همى كرييتوفيتها در اين منطقه حاكى از آن است كه منطقة مزبور كمتر تحت كشاورزى و كشت و زرع قرار گرفته است و اين نيز بدليل شيبهاى تند و ارتفاع زياد در اين منطقه است. از سوى ديخر حضور فراوان گونهاى جنس گون (Astragalus) با اشكال زيستى همى كريتوفيت نشاندهندة شرايط اقليم سرد در

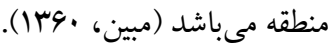

با توجه به GKY گونهُ گياهى موجود در منطقه و مقايسٔ آن با

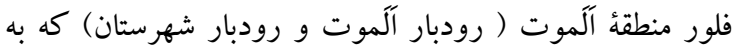

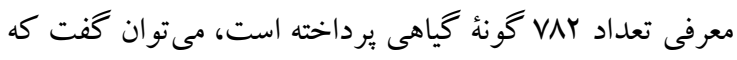

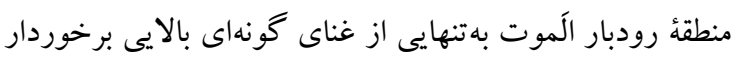

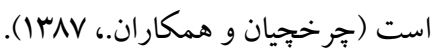
بهور كلى گياهان كاربردى اين منطقه را مىتوان به شش دسته تقسيم كرد كه شامل گياهان دارويى، انحصارى، سمى، مرتعى، كارئ علوفهاى و گياهان برمصرف در طب سنتى منطقه مىباشند. از

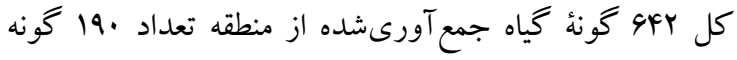

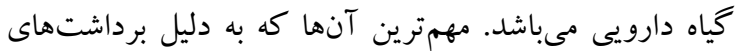
بschium (Ec) است كه در طب سنتى منطقه نيز فراوانى مصرف الى الى

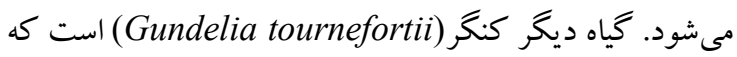
در فصل بهار به فراوانى برداشت مىشود و در بازارهاى سنتى قزوين به فروش مىرسد. كياهان سمى كه تعداد آنها WV كونه است بيشتر در دام و در

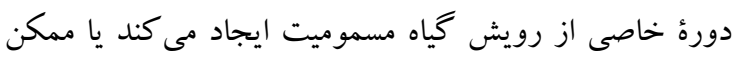

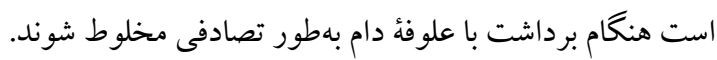

در زمينٔ گياهان مصرفى در طب سنتى منطقه مىتوان به گياه آوندول (Smyrnium cordifolium) اشاره كرد كه به دليل

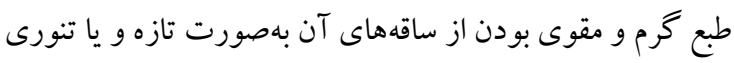
شده استفاده مى كنند. دربارة كاربردهاى گياهان در طب سنتى 


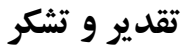
مراتب تشكر و قدردانى خود را از مسؤلان و همكاران محترم برّزوهشكدة گياهان دارويى جهاد دانشكاهى، مؤسسة تحقيقات

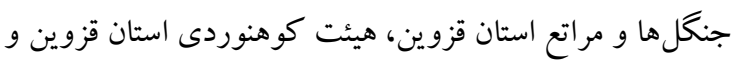

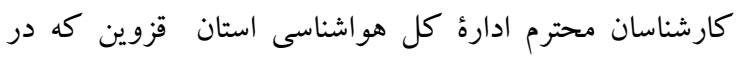
انجام اين تحقيق ما را صميمانه يارى كردند اعلام مى كنيم.

سيميسون، م.ج. بو إ. سيستماتيك گياهى. ترجمه فرخ قهرمانىنزاد و همكاران. - انتشارات خانه زيست شناسى. طرح توسعه و عمران حوزه آبريز شاهرود. ایدا. مطالعات راهبردى، جلد اول، جمهورى اسلامى ايران سازمان مديريت و برنامه

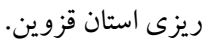

عصرى، ى. .ج"ا. گياهان مرتعى ايران، جلد اول. - انتشارات مؤسسه

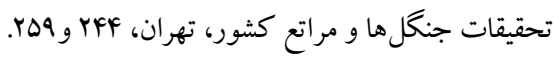
عصرى، ى. اqجا. گياهان مر تعى ايران، جلد دوم. - انتشارات مؤسسه

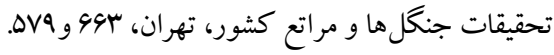

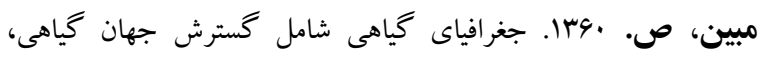

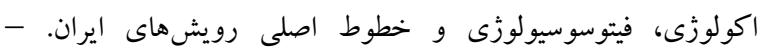

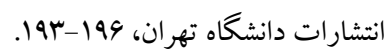
مظفريان، و. IVVA. فلور استان خوزستان. - مركز تحقيقات منابع طبيعى و امور دام خوزستان. مظفريان، و. rrV9. فلوراستان يزد. - انتشارات يزد. مظفريان، و. rیr|. درختان ودرختجههاى ايران. - نشر فرهنك مظفريان، و. اجوا. - شناخت گياهان دارويى و معطر ايران. - نشر

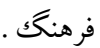

جنس مُلوسلا (Molucella) نيز كه فقط يك گونه گياه علفى يككساله به نام Molucella laevis در ايران دارد، تا به حال فقط از دشت مغان و اطر اف قصر شيرين جمع آورى شده بود نيز

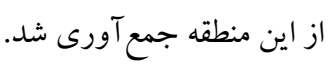

در انتها نامهاى علمى گياهان، شكلزيستى، عناصر رويشى و

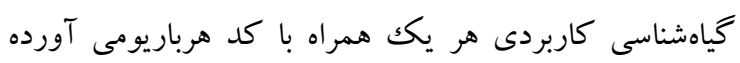

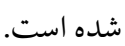

\section{منابع/References}

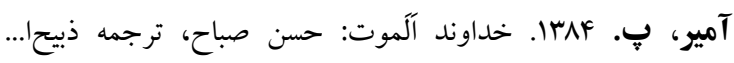
منصورى، جابٍ سوم. - انتشارات بدرقه جاويدان. جعفرى، ع. IrV9. كيتاشناسى ايران، دايره المعارف جغرافيايى ايران دانستىهاى ايران. - انتشارات گيتا شناسى. جورى، م.ح.، مهدوى، م. ودזו. شناسايى كاربردى گياهان

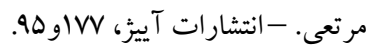

جرخجيان، م.م.، اكبرىنيا، ا. و ابطحى س.ف.

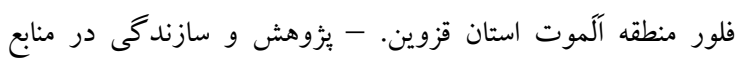

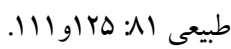

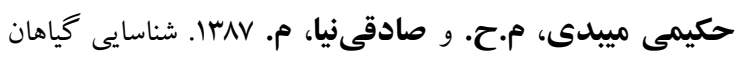

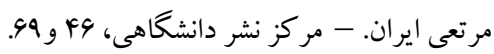

سازمان جغرافيايى نيروهاى مسلح (معاونت سنجش از دور و

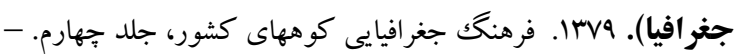
انتشارات سازمان جغر افيايى نيروهاى مسلح.

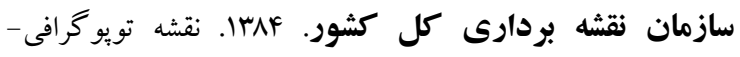

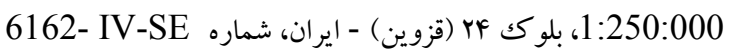

.6162- IV-SW, ستوده، م. بو كسا. قلاع اسماعيله در رشته كوههاى البرز. - انتشارات

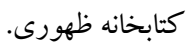

سيماى اقتصادى - اجتمايى شهرستان قزوين. ايرا. جمهورى اسلامى ايران سازمان مديريت و برنامدريزى استان قزوين. 


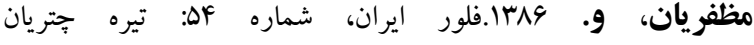

(Umbelliferae)

Bown, D. 1995. Encyclopedia of Herbs \& Their Uses. - Dorling Kindersleybook, London.

Davis, P.H. 1982. Flora of Turkey and the East Aegean. - Edinburgh University Press, Scotland.

Dewan, M.L. and Famouri, J. 1964. The Soils of Iran. - FAO, Italy.

Fleming T. 1998. PDR for Herbal Medicine. Medical Economics Company Montval, New Jersey.

Ghahremaninejad, F., Shabkhiz, R., Fereidounfar, S. 2012. A Florestic study on the weeds of wheat fields of Zanjan province, Iran. Pakistan Journal of Weed Sciences Research 18: 337-355.

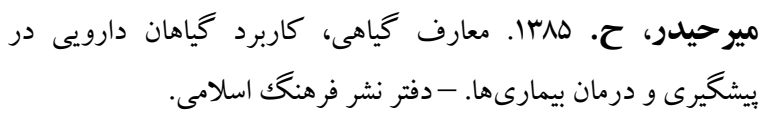

James, A. 1989. Handbook of Medicinal Herbs. Duke, CRC.

Jamzad, Z. and Assadi, M. 1984. New species of Nepeta and Ajuga in Iran. - Iran Jornal of Botany 2: 95-100.

Mozafarian, V.A., Ahvazi, M. and Charkhchian, M.M. 2008. A new species of the genus Lathyrus (Papilionaceae) from Iran. - The Iranian Journal of Botany 14: 7-9.

Rechinger, K.H. 1963-2005. Flora Iranica. Graz: Akademische Druck-und Verlasanstalt (1174). Vienna, Austria.

Townsend, C.C. 1966-1985. Flora of Iraq. Vol. 1-9. - Ministry of Agriculture and Agrarian Reform, Baghdad, Iraq.

Ahvazi, M., Mozaffarian, V. and Charkhchian, M.M. 2015. The investigation of flora in Roodbar Alamut region, Ghazvin, Iran. - Nova Biologica Reperta 2: 48-63.

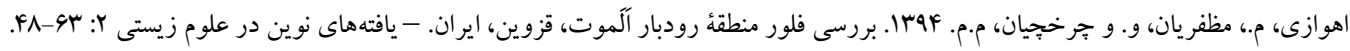


منطقهُ رويشى، شكلزيستى و گياهشناسى كاربردى كنار نام علمى هر گياه آورده شده است (علامت • نشاندهندة گياهانى است كه براى اولينبار در

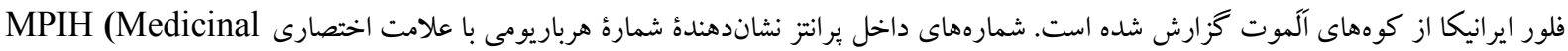
مىباشد.كياهان بدون شماره به دليل مناسب نبودن كيفيت آنها جهت جمع آورى و هرباريومى نمودن فقط با نام شناسايى كننده آورده شده است).

Appendix 1. List of plants in Rudbar Alamut region.

Life form, chorotype and applied botany are given for each plant. The sign [•] shows the plants that are reported of Alamut Mountain in Flora Iranica for the first time.

The numbers in brackets point out to the herbarium vocher specimens (MPIH: Medicinal Plants Institute Herbarium).

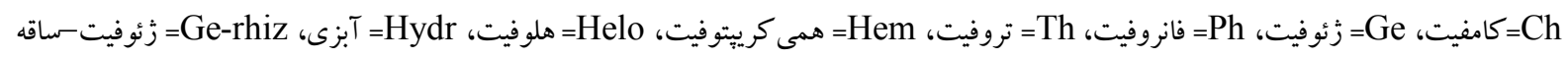

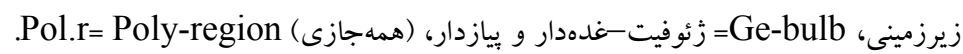

Saharo-Sindian = European-Siberian, Sa.Si= Irano-Turanian, Eu.Si= Ir.Tur

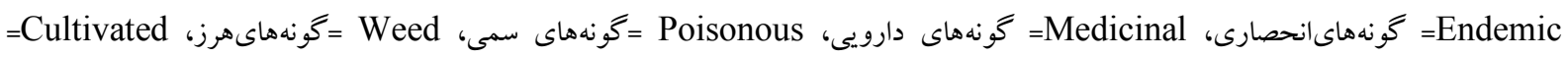
كونهاى زراعى و كاشتهشده، Forage= كو نهاى علوفهاى، Traditionalmedicine= طب سنتى منطقه)

Adianthaceae: Adianthum capillus-veneris L. [Pol. r; Ge-rhiz; M, T] (2994). Amaranthaceae: Amaranthus blitoides S.Watson [Pol. r, Th, M, W] (649); Amaranthus retroflexus L. [Pol. r; Th; F, P, W] (400). Amaryllidaceae: Ixiolirion tataricum (Pall.) Schult. \& Schult.f. [Ir. Tur; Ge-bulb] (645). Apiaceae: Astrodaucus orientalis Drude [Ir. Tur; Hem] (4001); Bilacunaria microcarpa (M.Bieb.) Pimenov \& V.N. Tikhom. [Ir. Tur; Hem; F] (s-n); Bunium cylindricum (Boiss. \& Hohen.) Drude [Ir. Tur -Sa. Si; Ge-bulb] (4002); Bunium persicum B.Fedtsch. [Ir. Tur; Ge-bulb; M, TF] (4003); Bupleurum falcatum L. [Ir. Tur; Hem; M] (401); Carum carvi L. [Ir. Tur - Eu. Si; Hem; M] (4004); Cervaria caucasica (M.Bieb.) Pimenov [Ir. Tur; Hem] (4005); Chaerophyllum bulbosum L. [Ir. Tur - Eu. Si; Ge-bulb] (4006); Chaerophyllum macropodum Boiss. [Ir. Tur; Hem; F] (402); Conium maculatum L. [Pol. r; Hem; P, M, F] (4007); Daucus carota L. [Pol. r; Hem; W, M] (3022); Diplotaenia cachrydifolia Boiss. [Ir. Tur; Hem; F] (402); Echinophora sibthorpiana Guss. [Ir. Tur; Hem; T, •] (4008); Eryngium billardieri Delar. [Ir. Tur; Hem; W, F] (4009); Eryngium caucasicum Trautv. [Ir. Tur - Eu. Si; Hem; W, F] (4010); Falcaria vulgaris Bernh. [Ir. Tur - Eu. Si - Sa. Si; Hem; M, W, T, •] (403); Ferula gummosa Boiss. [Ir. Tur; Hem; M, F] (405); Ferula ovina Boiss. [Ir. Tur; Hem; M, F] (406); Ferula persica Willd. [Ir. Tur; Hem; E, TF,] (407); Ferulago angulata Boiss. [Ir. Tur; Hem; M, F, •] (408); Grammosciadium platycarpum Boiss. \& Hausskn. ex Boiss. [Ir. Tur - Eu. Si; Hem; T, F, •] (409); Heptaptera anisoptera (DC.) Tutin [Ir. Tur; Hem] (4011); Heracleum persicum Desf. [Ir. Tur; Hem; M, TF,] (410); Kalakia stenocarpa (Bornm. \& Gauba) Alava [Ir. Tur; Th; E] (4012); Leutea petiolaris (DC.) Pimenov [Ir. Tur - Eu. Si; Hem, •] (412); Pimpinella affinis Ledeb. [Ir. Tur - Eu. Si; Hem; M] (414); Pimpinella tragioides (Boiss). Benth. \& Hook.f. ex. Drude [Ir. Tur; Hem; E, •] (415); Prangos uloptera DC. [Ir. Tur - Eu. Si; Hem; F] (s-n); Scandix iberica M.Bieb. [Ir. Tur - Sa. Si; Th; W] (3021); Scandix pecten-veneris L. [Ir. Tur - Eu. Si - Sa. Si; Th] (s-n); Scandix stellata Banks \& Soland [Ir. Tur - Eu. Si; Th] $(s-n)$; Sium sisaroideum DC. [Ir. Tur - Eu. Si; Ge-rhiz] $(s-n)$; Smyrnium cordifolium Boiss. [Ir. Tur; Hem; T] (4013); Stenotaenia nudicaulis Boiss. [Ir. Tur; Hem; E] (416); Torilis arvensis Link [Ir. Tur - Eu. Si; Th; W] (4014); Torilis leptophylla Rchb.f. [Ir. Tur - Eu. Si-Sa. Si; Th; W] (4015); Trachydium depressum Boiss. [Ir. Tur; Hem, •] (417); Zeravschanica aucheri (Boiss.) M.Pimen. [Ir. Tur Eu. Si; Hem] (4016); Zeravschanica membranacea (Boiss.) M. Pimen. [Ir. Tur - Eu. Si; Hem] (4017); 
Zosima absinthifolia Link [Ir. Tur; Hem] (4018); Zosima radians Boiss. \& Hohen. [Ir. Tur; Hem; E] (4019). Apocynaceae: Trachomitum venetum Woodson [Ir. Tur - Eu. Si; Ch] (643F); Vinca herbacea Waldst. \& Kit. [Ir. Tur- Eu. Si; Ge-rhiz] (418). Aristolochiaceae: Aristolochia bottae Jaub. \& Spach [Ir. Tur; Hem; P, W] (641). Asclepiadacea: Cynanchum acutum L. [Ir. Tur; Hem; M, P] (642); Vincetoxicum funebre Boiss. \& Kotschy [Ir. Tur; Hem; P] (419). -Asteraceae: Achillea aucheri Boiss. subs. glabra Hub.Mor. [Ir. Tur; Hem; E] (420); Achillea biebersteinii Afanasiev [Ir. Tur; Hem; M, F] (421); Achillea millefolium L. subsp. elbursensis Hub.Mor. [Ir. Tur; Hem ; M, E, T, F] (422); Achillea vermicularis Trin. [Ir. Tur; Hem] (423); Achillea wilhelmsii K.Koch [Ir. Tur - Sa. Si; Hem; M, W] (4020); Acroptilon repens (L.) DC. [Ir. Tur; Th; P, W] (4021); Arctium lappa L. [Ir. Tur - Eu. Si; Ge-rhiz; M, P, W] (424); Arctium minus (Hill) Bernh. [Ir. Tur - Eu. Si; Hem; M] (4022); Artemisia absinthium L. [Ir. Tur - Eu. Si; Hem; M] (4023); Artemisia splendens Willd. [Ir. Tur; Hem] (425); Carthamus lanatus L. [Ir. Tur; Th; W, M] (4024); Carthamus oxyacantha M.Bieb. [Ir. Tur; Th; M] (4025); Centaurea aucheri (DC.) Wagenitz subsp. elbursensis Wagenitz [Ir. Tur; Hem; F, •] (426); Centaurea behen L. [Ir. Tur; Hem; M, F] (4026); Centaurea depressa M.Bieb. [Ir. Tur; Th; M, F] (4027); Centaurea iberica Trev. ex Spreng. [Ir. Tur; Hem; F] (4028); Centaurea persica Boiss. [Ir. Tur; Hem; F] (4029); Centaurea pseudoscabiosa Boiss. \& Buhse [Ir. Tur; Hem; F] (4030); Centaurea pterocaula Trautv. [Ir. Tur; Hem; F] (4031); Centaurea rhizantha C.A.Mey. [Ir. Tur - Eu. Si; Hem; F] (427); Centaurea solstitialis L. [Ir. Tur; Th; F,P, M, •] (4032); Centaurea virgata Lam. [Ir. Tur - Eu. Si; Hem; F] (428); Cephalorrhynchus brassicifolius (Boiss.) Tuisl [Ir. Tur; Hem] (4033); Cephalorrhynchus takhtadzhianii (Sosn.) Kirp. [Ir. Tur - Eu. Si; Hem] (4034); Chardinia orientalis (L.) Kuntze [Ir. Tur; Th] (429); Cichorium intybus L. [Ir. Tur - Eu. Si- Sa. Si; Hem; M, F, W, P, •] (430); Cirsium congestum Fisch. \& C.A.Mey. ex DC. [Ir. Tur; Hem, •] (431); Cirsium hygrophilum Boiss. [Ir. Tur; Helo] (432); Cirsium lappaceum Lam. [Ir. Tur; Hem] (433); Cirsium obvallatum M.Bieb. [Ir. Tur; Hem; E] (434); Cnicus benedictus L. [Ir. Tur; Th; M] (4035); Conyza bonariensis (L.) Cronquist [Pol. r; Th] (4036); Cousinia archibaldii Rech.f. [Ir. Tur; Hem; E] (4037); Cousinia chamaepeuce Boiss. [Ir. Tur; Hem; E, •] (435); Cousinia crispa Jaub. \& Spach [Ir. Tur; Hem; E] (4038); Crepis elbursensis Boiss. [Ir. Tur; Hem; E, •] (s-n); Crepis foetida L. [Ir. Tur; Th] (4039); Crepis sancta (L) Babc. [Ir. Tur; Th] (4040); Crupina crupinastrum Vis. [Ir. Tur; Th] (4041); Echinops cephalotes DC. [Ir. Tur; Hem; EF,] (4042); Echinops elbursensis Rech.f. [Ir. Tur; Hem; EF,] (s-n); Echinops pungens Trautv. [Ir. Tur; Hem; M, F] (436); Erigeron acer L. [Ir. Tur Eu. Si; Hem; F] (437); Eupatorium cannabinum L. [Ir. Tur - Eu. Si; Hem; M] (4043); Garhadiolus angulosus Jaub. \& Spach [Ir. Tur - Eu. Si- Sa. Si; Th] (4044); Gundelia tournefortii L. [Ir. Tur; Hem; M, F, T] (2811); Helichrysum oligocephalum DC. [Ir. Tur; Hem; E, •] (439); Helichrysum psychrophilum Boiss. [Ir. Tur; Hem, •] (s-n); Heteropappus altaicus Novopokrov. [Ir. Tur - Eu. Si; Hem, •] (4045); Hieracium procerum Fr. [Ir. Tur - Eu. Si; Hem] (4046); Inula britannica L. [Ir. Tur; Hem; M, •] (4047); Inula salicina L. [Ir. Tur - Eu. Si; Hem] (440); Inula thapsoides Spreng. [Ir. Tur; Hem] (441); Jurinea marcocephala DC. [Ir. Tur; Hem; M, E] (4048); Jurinella microcephala (Boiss.) Wagenitz [Ir. Tur; Hem] (s-n); Koelpinia linearis Pall. [Ir. Tur; Th] (4049); Lactuca serriola L. [Ir. Tur - Eu. Si; Hem; W, F, M] (4050); Lapsana communis L. [Ir. Tur - Eu. Si; Hem; W] (442); Leontodon asperrimus (Willd.) Endl. [Ir. Tur; Hem] (443); Leontodon hispidus L. [Ir. Tur - Eu. Si- Sa. Si; Hem; FW,] (4051); Ligularia persica Boiss. [Ir. Tur; Hydr. ; E] (s-n); Onopordum acanthium L. [Ir. Tur - Eu. Si; Hem; F, M] (s-n); Onopordum leptolepis DC. [Ir. Tur Eu. Si- Sa. Si; Hem; F] (4052); Outreya carduiformis Jaub \& Spach [Ir. Tur; Th; E, •] (4053); Picnomon acarna (L.) Cass. [Ir. Tur; Th; M, •] (4045); Picris strigosa M.Bieb. [Ir. Tur; Hem; E, •] (444); Psychrogeton amorphoglossus (Boiss.) Novopokr. [Ir. Tur; Hem] (445); Scorzonera laciniata L. [Ir. Tur Eu. Si; Hem] (4055); Senecio othonnae M.Bieb. [Ir. Tur - Eu. Si; Hem] (4056); Senecio paucilobus DC. [Ir. Tur; Hem; E] (s-n); Senecio vernalis Hoppe ex DC. [Ir. Tur - Eu. Si; Th] (4057); Serratula latifolia Boiss. [Ir. Tur; Hem] (2810); Sonchus asper (L.) Hill. [Pol. r; Hem; W, M] (4058); Sonchus oleraceus L. [Ir. Tur Sa. Si; Hem; W, M] (446); Tanacetum canescens DC. [Ir. Tur; Hem] (447); Tanacetum kotschyi (Boiss) Grierson [Ir. Tur; Hem] (448); Tanacetum parthenium Schltz.Bip. [Ir. Tur - Eu. Si; Hem; M, •] (449); Tanacetum pinnatum Boiss. [Ir. Tur; Hem] (4059); Tanacetum polycephalum Schulz. Bip. [Ir. Tur; Hem] (4060); Tanacetum polycephalum Schulz.Bip. subsp. Duderanum(Boiss.) podlech. [Ir. Tur; Hem] (450); Taraxacum montanum (C.A.Mey.) DC. [Ir. Tur; Hem; F] (451); Tragopogon graminifolius DC. [Ir. Tur; Hem; W] (452); Tripleurospermum disciforme Sch.Bip. [Ir. Tur; Hem; M, T] (4061); Tussilago farfara L. [Ir. Tur - Eu. Si; Helo; M, W, T] (453); Xanthium spinosum L. [Pol. r; Th; M] (4062); Xanthium strumarium L. [Ir. Tur - Eu. Si; Th; M] (454); Xeranthemum inapertum M.Bieb. [Ir. Tur - Eu. Si; Th] (4063); Xeranthemum longepapposum Fisch. \& C.A.Mey. [Ir. Tur - Eu. Si; Th] (4064). Berberidaceae: Berberis 
integerrima Bunge [Ir. Tur; Ph; T, M] (455). Boraginaceae: Alkanna bracteosa Boiss. [Ir. Tur; Hem; M] (456); Anchusa italica Retz. [Ir. Tur - Eu. Si - Sa. Si; Hem; T, M] (457); Asperugo procumbens L. [Ir. Tur Eu. Si; Th] (692); Caccinia macranthera Brand [Ir. Tur; Hem; M] (678); Cerinthe minor L. [Ir. Tur - Eu. Si; Hem] (458); Echium amoenum Fisch \& C.A.Mey. [Ir. Tur; Hem; M, T] (3001); Gastrocotyle hispida Bunge [Ir. Tur - Sa. Si; Th] (677); Heterocaryum szovitsianum (Fisch. \& C.A.Mey.) A.DC. [Ir. Tur - Eu. Si - Sa. $\mathrm{Si}$; Th] (4065); Lappula microcarpa Gurke. [Ir. Tur; Hem] (676); Lithospermum arvense L. [Ir. Tur - Eu. Si; Th; F,W] (4066); Lithospermum officinale L. [Ir. Tur; Hem; M] (s-n); Moltkia coerulea Lehm. [Ir. Tur; Hem] (675); Nonnea caspica (Willd.) G.Don [Ir. Tur; Th] (4067); Onosma elwendicum Wettstein [Ir. Tur; Hem; E] (4068); Onosma sericeum Willd. [Ir. Tur; Hem] (679); Paracaryum rugulosum Boiss. [Ir. Tur - Eu. $\mathrm{Si}$ - Sa. Si; Hem] (4069); Rochelia disperma (L. F.) C. Koch [Ir. Tur - Eu. Si; Th] (4070); Solenathus circinnatus Ledeb. [Ir. Tur - Eu. Si; Hem] (4071); Trichodesma incanum Bunge [Ir. Tur; Hem] (459). Brassicaceae: Aethionema carneum (Banks \& Soland.) B.Fedtsch. [Ir. Tur; Th] (4072); Aethionema elongatum Boiss. [Ir. Tur - Eu. Si; Hem] (s-n); Aethionema grandiflorum Boiss. \& Hohen. [Ir. Tur; Hem; F] (460); Aethionema stenopterum Boiss. [Ir. Tur; Hem; E] (461); Alliaria petiolata (M.Bieb.) Cavara \& Grande [Ir. Tur - Eu. Si - Sa. Si; Th; M] (462); Alyssum linifolium Stephan ex Willd. [Ir. Tur; Th] (650); Anchonium elichrysifolium Boiss. [Ir. Tur; Hem] (463); Arabis aucheri Boiss. [Ir. Tur - Eu. Si; Hem] (658); Arabis nova Vill. [Ir. Tur - Eu. Si - Sa. Si; Th] (664); Barbarea plantaginea DC. [Ir. Tur; Ph] (660); Camelina rumelica Velen. subsp. rumelica [Ir. Tur - Eu. Si; Th] (464); Capsella bursa-pastoris (L.) Medik. [Pol. R; Hem; M, W, T, F] (465); Cardamine uliginosa M.Bieb. [Ir. Tur; Hem] (466); Cardaria draba (L) Desv. [Pol. r; Hem; W, M] (467); Clastopus erubescens Hausskn. [Ir. Tur; Hem; E] (s-n); Clastopus vestitus Boiss. [Ir. Tur; Hem; E] (468); Clypeola aspera Turill [Ir. Tur - Eu. Si - Sa. Si; Th] (4073); Clypeola jonthlaspi L. [Ir. Tur - Eu. Si; Th] (469); Conringia orientalis Andrz. ex DC. [Ir. Tur - Eu. Si; Th; M] (652); Crambe orientalis L. [Ir. Tur; Hem] (470); Descurainia sophia (L.) Webb ex Prantl [Ir. Tur - Eu. Si; Th; W, M, T] (670); Diplotaxis harra Boiss. [Ir. Tur - Sa. Si; Hem] (654); Draba aucheri Boiss. [Ir. Tur; Th, -] (671); Eruca sativa Mill. [Pol. r; Th; M, W] (672); Erysimum crassipes Fisch. \& C.A.Mey. [Ir. Tur; Hem] (668); Erysimum elbrusense Boiss. [Ir. Tur; Hem; E] (471); Euclidium syriacum (L.) R.Br. [Ir. Tur; Th] (666); Euclidium tenuissimum (Pall.) B.Fedtsch. [Ir. Tur; Th] (661); Fibigia suffruticosa (Vent.) Sweet [Ir. Tur; Hem] (597); Goldbachia laevigata (M.Beb.) DC. [Ir. Tur - Eu. Si; Th; M] (653); Graellsia saxifragifolia Boiss. [Ir. Tur; Hem] (655); Hesperis persica Boiss. [Ir. Tur; Hem] (667); Isatis cappadocica Desv. subsp. stenophylla (Bornm. \& Gauba) Hedge \& Lamond [Ir. Tur; Hem] (472); Lepidium perfoliatum L. [Ir. Tur; Th; M] (657); Malcolmia africana (L.) W.T.Aiton [Ir. Tur - Sa. Si; Th] (663); Matthiola ovatifolia Boiss. [Ir. Tur; Hem] (656); Nasturtium officinale W.T.Aiton [Ir. Tur - Eu. Si; Helo; P, M] (651); Parlatoria rostrata Boiss. \& Hohen. [Ir. Tur; Hem; E] (473); Physoptychis gnaphalodes Boiss. [Ir. Tur; Hem] (474); Pseudocamelina glaucophylla N.Busch [Ir. Tur; Hem; E] (475); Rapistrum rugosum (L.) All. [Ir. Tur - Eu. Si - Sa. Si; Th; P] (4074); Sinapis arvensis L. [Ir. Tur - Eu. Si - Sa. Si; Th; M] (662); Sisymbrium gaubae Rech. f. \& Bornm. [Ir. Tur - Eu. Si; Hem; W] (4075); Sisymbrium irio L. [Ir. Tur - Eu. $\mathrm{Si}$; Hem; M, F] (s-n); Thlaspi arvensis L. [Ir. Tur - Eu. Si; Th; M, W, FP,] (s-n); Thlaspi perfoliatum L. [Ir. Tur; Th] (659). Campanulaceae: Asyneuma amplexicaule Hand. Mazz. [Ir. Tur; Hem] (567); Asyneuma cichoriforme (Boiss.) Bornm. [Ir. Tur; Hem] (476); Campanula glomerata L. [Ir. Tur - Eu. Si; Hem] (s-n); Campanula involucrate Aucher ex A.DC. [Ir. Tur; Hem] (596); Campanula stevenii M.Bieb. [Ir. Tur; Hem] (477); Campanula stricta L. [Ir. Tur; Hem] (594); Mindium laevigatum (Vent.) Rech.f. \& Schiman-Czeika. [Ir. Tur; Hem] (s-n). Capparidaceae: Capparis spinosa L. [Ir. Tur; Ch; M, F, T] (636); Cleome coluteoides Boiss. [Ir. Tur; Hem] (4071); Cleome iberica DC. [Ir. Tur; Th] (478). Caprifoliaceae: Lonicera iberica M.Bieb. [Ir. Tur - Eu. Si; Ph; T, •] (479); Lonicera nummulariifolia Jaub. \& Spach [Ir. Tur; Ph] (s-n). Caryophyllaceae: Acanthophyllum glandulosum Bunge ex Boiss. [Ir. Tur; Ch] (700); Acanthophyllum microcephalum Boiss. [Ir. Tur; Ch, •] (2826); Arenaria gypsophiloides L. [Ir. Tur; Hem] (480); Arenaria gypsophiloides L. var. gypsophiloides L. [Ir. Tur; Hem] (480); Arenaria insignis Litv. [Ir. Tur; Hem] (481); Arenaria persica Boiss. [Ir. Tur; Hem; E]( s-n); Cerastium inflatum Link ex Sweet. [Ir. Tur; Th] (4077); Dianthus crinitus Sm. [Ir. Tur; Hem] (2857); Dianthus erythrocoleus Boiss. [Ir. Tur; Hem] (482); Dianthus orientalis Adams [Ir. Tur; Hem] (2858); Dianthus orientalis Adams subsp obtusisquameus (Boiss.) Rech.f. [Ir. Tur; Hem, •] (2827); Holosteum umbellatum L. [Pol. r; Th; M] (4078); Lepyrodiclis holosteoides Fenzl ex Fisch. \& C.A.Mey. [Ir. Tur - Eu. Si - Sa. Si; Th] (4079); Mesostemma kotschyanum (Fenzl) Vved. subsp. kotschyanum [Ir. Tur; Hem] (482); Minuartia lineate (Boiss.) Bornm. [Ir. Tur; Hem; E, •] (2831); Minuartia meyeri Bornm. [Ir. Tur; Th] (4080); Minuartia subtilis Hand.Mazz. [Ir. Tur - Sa. Si; Hem] (4081); 
Paronychia kurdica Boiss. [Ir. Tur - Eu. Si; Hem] (2832); Saponaria orientalis L. [Ir. Tur; Th; M] (4082); Scleranthus orientalis Rossler. [Ir. Tur; Th] (4083); Silene albescens Boiss. [Ir. Tur; Hem; E] (s-n); Silene chlorifolia Sm. [Ir. Tur; Hem] (484); Silene commelinifolia Boiss. [Ir. Tur; Hem] (4084); Silene commelinifolia Boiss. var. commelinifolia [Pol. r; Hem] (485); Silene coniflora Nees ex Otth [Ir. Tur; Th] (sn); Silene conoidea L. [Pol. r; Th; M] (4085); Silene latifolia Poir. [Ir. Tur - Eu. Si; Hem] (2830); Silene latifolia Poir. subsp. alba (Mill.) Greuter \& Burdet [Ir. Tur - Eu. Si; Hem] (4086); Silene marschallii C.A.Mey. [Ir. Tur - Sa. Si; Hem] (4087); Silene spergulifolia M.Bieb. [Ir. Tur; Hem] (2828); Silene viscosa Pers. [Ir. Tur - Eu. Si; Hem] (486); Stellaria holostea L. [Ir. Tur - Eu. Si; Hem] (2829); Stellaria media (L.) Vill. [Pol. r; Th; M, W] (487); Vaccaria oxyodonta Boiss. [Ir. Tur - Eu. Si; Th] (2825). Chenopodiaceae: Camphorosma monspeliaca L. [Ir. Tur - Eu. Si; Ch; M, F] (4088); Chenopodium album L. [Pol. r; Th; M, W, F,P T] (s-n); Chenopodium botrys L. [Pol. r; Th; W, F, M, T] (4089); Chenopodium foliosum (Moench) Asch. [Pol. r; Th; W, F] (739); Chenopodium murale L. [Ir. Tur - Eu. Si; Th; M] (4090); Chenopodium vulvaria L. [Ir. Tur - Eu. Si; Th; M] (4091); Noaea mucronata (Forssk.) Asch. \& Schweinf. [Ir. Tur; Ch] (4092). Cistaceae: Helianthemum salicifolium (L.) Mill. [Ir. Tur; Th] (598). Convolvolaceae: Convolvulus arvensis L. [Pol. r; Ge-rhiz; M, W] (488); Convolvulus commutatus Boiss. [Ir. Tur - Eu. Si; Hem] (489). Cornaceae: Cornus australis C.A.Mey. [Ir. Tur - Eu. Si; Ph] (738); Cornus mas L. [Ir. Tur - Eu. Si; Ph; M] $(s-n)$. Corylaceae: Corylus avellana L. [Pol. r; Ph; M] $(s-n)$. Crassulaceae: Sedum tenellum M.Bieb. [Ir. Tur - Eu. Si; Th] (4093). Cucurbitaceae: Bryonia monoica Aitch \& Hemsl. [Ir. Tur - Eu. Si; Hem; E] (4094). Datiscaceae: Datisca cannabina L. [Ir. Tur-Eu. Si; Hem M; ] (4095). Cupressaceae: Juniperus excelsa M.Bieb. [Ir. Tur; Ph; M] (490). Cyperaceae: Bolboschoenus maritimus (L.) Palla [Pol. r; Ge-bulb] (4234); Carex demissa Hornem. [Pol. r; Hem] (4235); Carex divulsa Stokes [Eu. Si - Sa. Si; Hem] (4236); Carex stenophylla Wahlenb. [Ir. Tur; Ge-rhiz] (4237); Cyperus longus L. [Ir. Tur; Hem; M] (4238); Scirpoides holoschoenus (L.) Sojak [Ir. Tur; Hem] (4239). Dipsacaceae: Cephalaria procera Fisch. \& AveLall. [Ir. Tur; Hem; W] (s-n); Dipsacus strigosus willd. ex Roem. \& Schult. [Ir. Tur; Hem] (492); Pterocephalus canus Coult. ex DC. [Ir. Tur; Hem; E] (493); Pterocephalus plumosus Coult. [Ir. Tur - Eu. Si; Th] (4096); Scabiosa calocephala Boiss. [Ir. Tur; Th] (4097); Scabiosa olivieri Coult. [Ir. Tur - Eu. Si; Th] (4098). Elaeagnaceae: Elaeagnus angustifolia L. [Ir. Tur; Ph; M, T] (4099); Hippophae rhamnoides L. [Ir. Tur - Eu. Si; Ph; T, M] (494). Ephedraceae: Ephedra major Host. [Ir. Tur - Eu. Si; Ph; M] (2996). Equisetaceae: Equisetum arvenseL. [Ir. Tur - Eu. Si, Hem, W,P,M](691); Equisetum ramosissimum Desf. [Ir. Tur - Eu. Si, Hem](4000). Euphorbiaceae: Andrachne telephioides L. [Ir. Tur; Hem] (701); Chrozophora hierosolymitana Spreng. [Pol. r; Th] (s-n); Chrozophora tinctoria (L.) A.Juss. [Ir. Tur - Eu. Si; $\mathrm{Th}$ ] (s-n); Euphorbia aucheri Boiss. [Ir. Tur; Hem] (s-n); Euphorbia chieradenia Boiss. \& Hohen. [Ir. Tur; Hem; T] (4100); Euphorbia denticulata Lam. [Ir. Tur; Hem] (4101); Euphorbia helioscopia L. [Ir. Tur - Eu. $\mathrm{Si}$ - Sa. Si; Th; WP,] (4102); Euphorbia myrsinites L. [Ir. Tur - Eu. Si; Hem] (4103); Euphorbia petiolata Banks \& Soland. [Ir. Tur; Th] (s-n); Euphorbia szovitsii Fisch. \& C.A.Mey. [Ir. Tur; Th] (4104); Euphorbia virgata Waldst. \& Kit. [Ir. Tur - Eu. Si; Hem] (4105). Fumariaceae: Fumaria asepala Boiss. [Ir. Tur - Eu. $\mathrm{Si}$; Th; T] (638); Fumaria vaillantii Loisel. [Ir. Tur - Eu. Si; Th; M] (2821). Gentianaceae: Centaurium minus Moench. [Ir. Tur - Eu. Si; Th; M] (516); Gentiana septemfida pall. [Ir. Tur; Hem] (517); Swertia longifolia Boiss. [Ir. Tur; Helo] (518). Geraniaceae: Biebersteinia multifida DC. [Ir. Tur; Ge-bulb; M] (4106); Erodium cicutarium (L.) L Her. ex Aiton [Ir. Tur - Eu. Si; Th; M, WP,] (4107); Geranium lucidum L. [Ir. Tur - Eu. Si - Sa. Si; Th] (4108); Geranium pyrenaicum Burm. f. [Ir. Tur - Eu. Si - Sa. Si; Hem] (4109); Geranium tuberosum L. [Ir. Tur; Ge-bulb] (4110). Hypericaceae: Hypericum helianthemoides (Spach) Boiss. [Ir. Tur; Hem] (2996); Hypericum perforatum L. [Ir. Tur - Eu. Si - Sa. Si; Hem; M, FP,] (519); Hypericum scabrum L. [Ir. Tur; Hem; T] (520). Iridaceae: Gladiolus atroviolaceus Boiss. [Ir. Tur; Ge-bulb; T] (4240); Iris barnumae Foster \& Baker [Ir. Tur; Ge-bulb] (4241). Juglandaceae: Juglans regia L. [Ir. Tur - Eu. Si; Ph; M, T] (4111). Juncaceae: Juncus articulatus L. [Pol. r; Hem] (4242); Juncus inflexus L. [Pol. r; Hem; P] (4243). Lamiaceae: Acinos graveolens Link. [Ir. Tur - Eu. Si; Th] (4112); Ajuga chamaecistus Ging. ex Benth. [Ir. Tur; Hem; M] (4113); Dracocephalum kotschyi Boiss. [Ir. Tur; Hem; M, E, T] (521); Dracosephalum multicaule Montbr. \& Auch. [Ir. Tur; Hem; M] (522); Eremostachys laciniata (L.) Bunge in Ledeb. [Ir. Tur; Hem] (4114); Lallemantia iberica (Stev.) Fisch. \& C.A.Mey. [Ir. Tur - Eu. Si; Th; M] (4115); Lallemantia peltata (L.) Fisch. \& C.A.Mey [Ir. Tur; Th] (4116); Lallemantia royleana (Benth. in Wall.) Benth. in DC. in DC. [Ir. Tur; Th] (s-n); Lamium album L. [Ir. Tur - Eu. Si; Hem; W, M] (751); Lamium amplexicaule L. [Ir. Tur - Eu. Si; Th; W, F, M] (523); Leontodon stenocalathius Rech.f. [Ir. Tur; Hem; E] (4117); Leonurus cardiaca L. [Ir. Tur - Eu. Si; Hem; M] (524); Marrubium astracanicum 
Jacq. [Ir. Tur - Eu. Si; Hem] (2800); Marrubium cuneatum Russel [Ir. Tur; Hem, •] (4118); Mentha longifolia (L.) Hudson [pol. r; Helo; M, T] (525); Molusella laevis L. [Ir. Tur; Th] (2807); Nepeta crassifolia Boiss. \& Buhse [Ir. Tur; Hem; M, E] (2806); Nepeta denudata Benth. [Ir. Tur; Hem; E] (2805); Nepeta fissa C.A.Mey. [Ir. Tur; Hem] (526); Nepeta pogonesperma Jamzad \& Assadi [Ir. Tur; Hem; E, T] (527); Nepeta rasemosa Lam. [Ir. Tur - Eu. Si; Hem; M] (528); Nepeta saccharata Bunge [Ir. Tur; Th; E] (s-n); Phlomis olivieri Benth. [Ir. Tur; Hem; E] (529); Prunella vulgans L. [Ir. Tur - Eu. Si; Hem; W, M] (2804); Salvia aethiopis L. [Ir. Tur - Eu. Si; Hem; M] (2803); Salvia hypoleuca Benth. in DC. [Ir. Tur; Hem; E,M] (s-n); Salvia limbata C.A.Mey. [Ir. Tur; Hem] (2802); Salvia multicaulis Vahl. [Ir. Tur - Eu. Si; Hem] (2801); Salvia nemorosa L. [Ir. Tur - Eu. Si; Hem, •] (s-n); Salvia reuterana Boiss. [Ir. Tur; Hem; E] (530); Salvia sclarea L. [Ir. Tur - Eu. Si; Hem; M] (531); Salvia syriaca L. [Ir. Tur - Eu. Si; Hem; M] (4119); Salvia verticillata L. [Ir. Tur - Eu. Si; Hem] (532); Salvia virgata Jacq. [Ir. Tur - Eu. Si; Hem; M, •] (4120); Salvia xantocheila Boiss. ex Benth. in DC. [Ir. Tur - Eu. Si; Hem] (533); Scutellaria pinnatifida A. Hamilt. [Ir. Tur - Eu. Si; Hem] (4121); Sideritis montana L. [Ir. Tur - Eu. Si; Th] (4122); Stachys fruticulosa M.B. [Ir. Tur; Ch] (4123); Stachys inflata Benth. [Ir. Tur; Hem; M] (4124); Stachys lavandulifolia Vahl. [Ir. Tur; Hem; M, TF,] (534); Stachys pubesces Ten. [Ir. Tur; Hem] (535); Teucrium orientale L. [Ir. Tur; Hem; M] (4125); Teucrium polium L. [Ir. Tur - Eu. Si - Sa. Si] (536); Thymus pubescens Boiss. \& Kotschy ex Celak. [Ir. Tur; Hem; TF,] (4126); Ziziphora capitata L. subsp. orientalis Samuelsson ex Rech. f. [Ir. Tur - Eu. Si; Th; T] (sn); Ziziphora capitata L. [Ir. Tur - Eu. Si; Th; T] (537); Ziziphora clinopodioides Lam. [Ir. Tur; Hem; M] (538); Ziziphora tenuior L. [Ir. Tur; Th; M, T] (4127). Liliaceae: Allium akaka Gmel. ex Roem. \& Schult. [Ir. Tur; Ge-bulb; M] (2882); Allium minutiflorum Regel [Ir. Tur; Ge-bulb; E] (4244); AlliumscabriscapumBoiss. [Ir. Tur; Ge-bulb] (s-n); Allium schoenoprasum L. [Pol. r; Ge-bulb] (539); Bellevalia glauca Kunth [Ir. Tur; Ge-bulb] (4245); Colchicum kotschyi Boiss. [Ir. Tur; Ge-bulb] (681); Colchicum speciosumsteven [Ir. Tur - Eu. Si; Ge-bulb] (4246); Gagea gageoides (Zucc.) Vved. [Ir. Tur; Gebulb] (4247); Gagea rigida Boiss. \& Spruner [Ir. Tur - Eu. Si; Ge-bulb] (4248); Muscaria longipes Boiss. [Ir. Tur - Eu. Si; Ge-bulb] (4249); Muscaria neglectum Guss. ex Ten. [Ir. Tur - Eu. Si; Ge-bulb] (4250); Ornithogalum cuspidatum Bertol. [Ir. Tur - Eu. Si; Ge-bulb] (4251); Tulipa biflora Pall. [Ir. Tur; Ge-bulb] (4252); Tulipa biebersteiniana Schult.f. [Ir. Tur; Ge-bulb] (4253); Tulipa Montana Lindl. [Ir. Tur; Ge-bulb] (4254); Tulipa Montana Lindl var chrysantha (Boiss) Wendelbo ex Rech.f. [Ir. Tur; Ge-bulb] (4255); Tulipa systola Stapf. [Ir. Tur; Ge-bulb] (4256). Linaceae: Linum album Kotschy. ex Boiss. [Ir. Tur; Hem; M] (4128); Linum catharticum L. [Ir. Tur; Th; M] (540). Lythraceae: Lythrum salicaria L. [Ir. Tur - Eu. Si Sa. Si; Hem; M] (4129). Malvaceae: Alcea longipedicellata I.Riedl. [Ir. Tur; Hem; E] (s-n); Alcea sulphurea Alef. [Ir. Tur; Hem] (4130); Malva neglecta Wallr. [Ir. Tur - Eu. Si - Sa. Si; Hem; M, W, •] (541); Malva sylvestris L. [Ir. Tur - Eu. Si; Hem; M, W] (4131). Moraceae: Ficus carica L. [Ir. Tur - Eu. $\mathrm{Si}$ Ph; M] (s-n); Morus alba L. [Ir. Tur - Eu. Si - Sa. Si; Ph; M] (4132); Morus nigra L. [Ir. Tur - Eu. Si $\mathrm{Sa} . \mathrm{Si} ; \mathrm{Ph} ; \mathrm{M}$ ] (4133). Oleaceae: Fraxinus angustifolia Vahl [Ir. Tur - Eu. Si - Sa. Si; $\mathrm{Ph} ; \mathrm{C}$ ] (s-n). Onagraceae: Epilobium algidum M.Bieb. [Ir. Tur; Hem] (s-n); Epilobium hirsutum L. [Ir. Tur - Eu. Si - Sa. Si; Hem; M] (542). Orchidaceae: Dactylorhiza umbrosa (Kar. \& Kir.) Nevski [Ir. Tur - Eu. Si; Ge-bulb] (4257); Epipactis veratrifolia Boiss. \& Hohen. [Ir. Tur - Eu. Si; Ge-bulb] (4258). Orobanchaceae: Orobanche kotschyi Reut. [Ir. Tur- Sa. Si; Ge-rhiz] (745). Papaveraceae: Glaucium contortuplicatum Boiss. [Ir. Tur; Hem] (4134); Papaver argemone L. [Ir. Tur - Eu. Si - Sa. Si; Th; W] (4135); Papaver dubium L. [Ir. Tur; Th; W, M] (4136); Papaver fugax Poir. [Ir. Tur - Eu. Si - Sa. Si; Hem] (2808); Papaver rhoeas L. [Ir. Tur - Eu. Si - Sa. Si; Hem; M, W, F, P] (543); Roemeria hybrida (L.) DC. [Ir. Tur - Eu. Si Sa. Si; Th; W] (4137); Roemeria refracta DC. [Ir. Tur; Th] (4138). Papilionaceae: Alhagi camelorum Fish. [Ir. Tur; Hem; W, F] (4139); Argyrolobium trigonelloides Jaub. \& Spach. [Ir. Tur; Hem] (4140); Astragalus jodotropis Boiss. [Ir. Tur; Ch] (496); Astragalus ochrochlorus Boiss. \& Hohen. [Ir. Tur; Ch] (502); Astragalus aureus Wild. [Ir. Tur; Ch] (495); Astragalus michauxianus Boiss. [Ir. Tur; Ch] (4141); Astragalus kirrindicus Boiss. \& Noe. [Ir. Tur; Hem; P] (4142); Astragalus yessenii Bunge, Mem. [Ir. Tur; Hem] (4143); Astragalus submitis Boiss. \& Hausskn. [Ir. Tur; Hem; E] (497); Astragalus aegobrromus Boiss \& Hohen. [Ir. Tur; Hem; F] (4144); Astragalus dipelta Bunge [Ir. Tur - Eu. Si; Th] (4145); Astragalus campylathus Boiss. [Ir. Tur; Hem; E] (4146); Astragalus lagopoides Lam. [Ir. Tur; Ch] (4147); Astragalus persicus (DC.) F.\& M. [Ir. Tur; Ch; E] (4148); Astragalus askius Bunge. [Ir. Tur; Hem; F, E] (4149); Astragalus curvirostris Boiss. [Ir. Tur; Hem; F] (498); Astragalus iranicus Bunge. [Ir. Tur; Hem; E] (4150); Astragalus effusus Bunge. [Ir. Tur; Hem; F, E] (4151); Astragalus oxyglottis Stev. \& M.Beib. [Ir. Tur; Th; P] (503); Astragalus gossypinus Fisch. [Ir. Tur; Ch; M, F] (4152); Astragalus microcephalus Willd. [Ir. Tur; 
Ch; M] (499); Astragalus compactus Reiche. [Ir. Tur; Ch] (500); Astragalus rhodosemius Boiss. \& Hausskn. [Ir. Tur; Ch; E] (504); Astragalus capito Boiss \& Hohen. [Ir. Tur; Hem; E] (501); Cicer spiroceras Jaub. \& Spach [Ir. Tur; Hem; E] (506); Cicer tragacanthoides Jaub. \& Spach [Ir. Tur - Eu. Si; Hem; E] (507); Colutea buhsei (Boiss) Shapar. [Ir. Tur; Ph, •] (4153); Coronilla orientalis Mill. [Ir. Tur - Eu. Si; Hem] (4154); Coronilla varia L. [Ir. Tur - Eu. Si; Hem; W, M, F, •] (508); Glycyrrhiza glabra L. [Ir. Tur - Eu. Si; Ge-rhiz; M] (4155); Lathyrus alamutensis Mozaff., Ahvazi \& Charkhchian [Ir. Tur; Hem; E] (505); Lathyrus aphaca L. [Pol. r; Hem; F, M] (2879); Lathyrus inconspicuous L. [Ir. Tur - Eu. Si; Th] (4156); Lens culinaris Mediks. [Pol. r; Th; M] (4157); Lens orientalis (Boiss.) Popov [Pol. r; Th] (4158); Lotus corniculatus L. [Ir. Tur - Eu. Si; Hem; P, M, F, •] (4159); Lotus gebelia Vent. [Ir. Tur; Hem; W, F] (509); Medicago lupulina L. [Ir. Tur - Eu. Si; Hem; W, F] (510); Medicago minima (L.) L. ex Bartal. [Ir. Tur - Eu. $\mathrm{Si}$ - Sa. Si; Th; F] (s-n); Medicago rigidula (L.) All. [Ir. Tur - Eu. Si; Th; F] (2878); Melilotus albus Desr. [Ir. Tur - Eu. Si; Hem ; FW, M,] (2880); Melilotus officinalis (L) Pall. [Ir. Tur - Eu. Si; Hem; W, M] (511); Onobrychis cornuta (L.) Desv. [Ir. Tur; Ch; F, •] (512); Onobrychis haussknechtii Boiss. [Ir. Tur; Hem; F] (4160); Ononis spinosa L. [Ir. Tur; Ch; W, F, M] (4161); Oxytropis kotschyana Boiss. \& Hohen. [Ir. Tur; Hem; E] (4162); Oxytropis szovitsii Boiss. \& Buhse [Ir. Tur; Hem; E] (4163); Pisum formosum Alef. [Ir. Tur - Eu. Si; Hem; E] (513); Pisum sativum L. [Ir. Tur; Th; F, M] (s-n); Sophora alopecuroides L. [Ir. Tur; Hem; M, W] (4164); Trifolium fragiferum L. [Pol. r; Hem; FP, •] (4165); Trifolium pratense L. [Ir. Tur - Eu. Si; Hem; FP, M,] (514); Trifolium repens L. [Ir. Tur - Eu. Si; Hem; P,M,W,F] (515); Trigonella coerulescens (M.Bieb.) Halàcsy [Ir. Tur - Eu. Si; Th] (4166); Trigonella elliptica Boiss. [Ir. Tur; Hem; F] (4167); Trigonella monantha C.A.Mey. [Ir. Tur; Th] (4168); Trigonella monspeliaca L. [Ir. Tur - Eu. Si; Th] (4169); Trigonella spruneriana Boiss. [Ir. Tur - Eu. Si; Th] (4170); Vicia ciceroidea Boiss. [Ir. Tur; Hem] (s-n); Viciaervilia Willd. [Ir. Tur - Eu. Si - Sa. Si; Hem] (4171); Vicia monantha Retz. [Ir. Tur - Eu. Si; Hem] (4172); Vicia persica Boiss. [Ir. Tur; Hem; P] (4173); Vicia sativa L. [Pol. r; Th; M] (4174); Vicia villosa Roth [Ir. Tur - Eu. Si; Hem; F] (s-n). Plantaginaceae: Plantago atrataHoppe [Ir. Tur - Eu. Si; Hem] (544); Plantago lanceolata L. [Ir. Tur - Eu. Si - Sa. Si; Hem; M, W] (545); Plantago major L. [Ir. Tur - Eu. Si; Hem; M, W, T] (546). Plumbaginaceae: Acantholimon erinaceum (Jaub. \& Spach) Lincz. [Ir. Tur; Ch; F, •] (547). Poaceae: Aegilops columnaris Zhuk. [Ir. Tur - Eu. Si; Th] (4259); Aegilopstriuncialis L. [Ir. Tur Eu. Si; Th; F] (4260); Agropyron intermedium (Host) P.Beauv. [Ir. Tur; Hem] (4261); Agropyron leptourum (Nevski) Grossh. [Ir. Tur - Eu. Si; Hem, •] (4262); Agropyron longe-aristatum (Boiss) Boiss. [Ir. Tur; Hem, -] (548); Agropyron pectiniforme Roem. \& Schult. [Ir. Tur - Eu. Si; Hem] (4263); Agropyron podperae Nab. [Ir. Tur; Hem] (4264); Agropyron repens (L.). P.Beauv. [Ir. Tur - Eu. Si - Sa. Si; Ge-rhiz; W, M] (4265); Agropyron tauri Boiss. \& Balansa [Ir. Tur; Hem] (549); Agropyron trichophorum K.Richt. [Ir. Tur Eu. Si - Sa. Si; Hem; F] (4266); Alopecurus apiatus Ovcz. [Ir. Tur; Hem; F] (4267); Alopecurus vaginatus Pall. [Ir. Tur; Hem; F] (550); Arrhenatherum kotschyi Boiss. [Ir. Tur; Ge-rihz; F] (4268); Avena clauda Durieu [Ir. Tur - Eu. Si - Sa. Si; Th] (4269); Avena wiestii Steud. [Ir. Tur - Sa. Si; Th] (4270); Boissiera squarrosa (Banks \& Soland.) Nevski [Ir. Tur; Th; F] (4271); Bothriochloa ischaemum (L.) Keng [Pol. r; Hem; F] (551); Brachypodium pinnatum (L.) P.Beauv. [Ir. Tur - Eu. Si; Hem; F] (4272); Brachypodium sylvaticum (L.) P.Beauv. [Ir. Tur - Eu. Si; Hem; W] (552); Bromus cappadocicus Boiss \& Balansa [Ir. Tur Eu. Si; Hem] (s-n); Bromus danthoniae Trin. ex C.A.Mey. [Ir. Tur; Th] (553); Bromus sterilis L. [Ir. Tur; Th] (4273); Bromus tectorum L. [Pol. r; Th] (4274); Calamagrostis pseudophragmites (Hah.F.) Koel. [Ir. Tur - Eu. Si - Sa. Si; Hem] (554); Cynodon dactylon (L.) Pers. [Pol. r; Ge-rhiz; F, W, P, M] (555); Dactylis glomerata L. [Ir. Tur - Eu. Si; Hem; W, F] (556); Deschampsia caespitosa (L.) P.Beauv. [Pol. r; Ch; F] (4275); Deyeuxia parsana Bor. [Ir. Tur; Hem; E] (557); Echinaria capitata (L.) Desf. [Ir. Tur - Eu. Si - Sa. Si; Th] (4276); Echinochloa crus-galli (L.) P. Beauv. [Ir. Tur - Eu. Si; Th; W,P,M, F] (4277); Eremopoa persica (Trin.) Roshev. [Ir. Tur - Eu. Si - Sa. Si; Th; F] (4278); Eremopyrum confusum Melderis [Ir. Tur; Th] (4279); Festuca ovina L. [Ir. Tur - Eu. Si - Sa. Si; He; F] (560); Heteranthelium piliferum (Banks \& Soland.) Hochst. [Ir. Tur - Eu. Si - Sa. Si; Th] (4280); Hordeum bulbosum L. [Ir. Tur - Sa. Si; Ge-rhiz; F] (4281); Hordeummarinum Huds. [Ir. Tur; Th; F] (561); Hordeumviolaceum Boiss. \& Hohen. [Ir. Tur; Hem; F] (4282); Lolium rigidum Gaudin [Ir. Tur - Eu. Si; Th; P, F] (4283); Lophochloa phleoides (Vill.) Rchb. [Ir. Tur - Eu. Si; Th] (4284); Melica jacquemontii Decne. [Ir. Tur; Hem; F] (4285); Oryzopsis lateralis Stapf in Hook.f. [Ir. Tur; Hem] (562); Pennisetum orientale Rich [Ir. Tur - Sa. Si; Hem; F] (4286); Phragmites australis (Cay.) Trin. ex Steud. [Ir. Tur - Eu. Si; Ch; F, M] (563); Poa araratica Trautv. [Pol. r; Hem] (4287); Poa bulbosa L. [Ir. Tur- Sa. Si; Ge-rhiz; F] (564); Poa nemoralis L. [Ir. Tur - Eu. Si; Hem; W, F] (4288); Polypogon fugax Nees ex Steud. [Ir. Tur - Eu. Si; Hem] (4289); Psathyrostachys fragilis (Boiss). 
Nevski. [Ir. Tur-Sa. Si; Hem; F] (565); Saccharum ravennae (L.) Murray [Ir. Tur- Sa. Si; Ge-rhiz] (4290); Sclerochloa dura (L.) P. Beauv. [Ir. Tur - Eu. Si; Th] (4291); Setaria viridis (L.) P. Beauv. [Ir. Tur; Th; FW, M,] (4292); Sorghum halepense (L.) Pers. [Ir. Tur; Ge-rhiz; M] (2885); Stipa hohenackeriana Trin. \& Rupr. [Ir. Tur; Hem; F] (4293); Taeniatherum crinitum (Schreb.) Nevski [Ir. Tur; Th; F] (566); Trisetum flavescens (L.) P. Beauv. [Ir. Tur - Eu. Si - Sa. Si; Hem; F] (567); Vulpia ciliata Dumort. [Ir. Tur - Eu. Si - Sa. Si; Th] (4294). Podophyllaceae: Bongardia chrysogonum Boiss. [Ir. Tur; Ge-bulb; M] (4175). Polygonaceae: Atraphaxis spinosa L. [Ir. Tur; Ph; M] (745); Oxyria digyna Hill. [Ir. Tur - Eu. Si; Hem; F, •] (568); Polygonum alpestre C.A.Mey. [Ir. Tur; Hem] (569); Polygonum aviculare L. [Pol. r; Th; W, M] (s-n); Polygonum bistorta L. [Ir. Tur - Eu. Si; Helo; M] (570); Polygonum lapathifolium L. [Ir. Tur - Eu. Si; Helo; W, M] (4176); Polygonum patulum M.Bieb. [Ir. Tur - Eu. Si - Sa. Si; Th] (4177); Polygonum persicaria L. [Pol. r; Helo; W, M] (4178); Polygonum rottboellioides Jaub. \& Spach [Ir. Tur; Th] (s-n); Pteropyrum aucheri Jaub. \& Spach [Ir. Tur; Ph] (4179); Rheum ribes L. [Ir. Tur; Hem; M, T] (4180); Rumex dentatus L. [Pol. r; Hem] (s-n); Rumex elbursensis Boiss. [Ir. Tur; Hem; T, M] (4181); Rumex pulcher L. [Ir. Tur - Eu. Si; Hem; M] (571); Rumex scutatus L. [Ir. Tur - Eu. Si; Hem; M] (572). Portulacaceae: Portulaca oleracea L. [Pol. r; Th; W, F, P, M] (4182). Primulaceae: Androsace maxima L. [Ir. Tur; Th] (4183); Primula auriculata Lam. [Ir. Tur - Eu. Si; Hem] $(s-n)$. Ranunculaceae: Adonis aestivalis L. [Ir. Tur; Th; W, M] (4184); Aquilegia olympica Boiss. [Ir. Tur - Eu. Si; Hem] (4185); Ceratocephalus falcatus (L.) Pers. [Ir. Tur - Eu. Si; Th] (4186); Clematis orientalis L. [Ir. Tur; Ph; M] (4187); Delphinium saniculifolium Boiss. [Ir. Tur; Ge-rhiz] (4188); Ficaria kochii (Ledeb). Iranshahr \& Rech.f. [Ir. Tur; Ge-rhiz; M] (4189); Ranunculus arvensis L. [Ir. Tur; Th; F, M] (4190); Ranunculus kotschyi Boiss. [Ir. Tur; Hem] (4191). Resedaceae: Reseda lutea L. [Ir. Tur - Eu. Si; Hem; W, M] (573). Rhamnaceae: Paliurus spina-christi Mill. [Ir. Tur Eu. Si; Ph; M, T] (4192); Rhamnus pallasii Fisch. \& C.A.Mey. [Ir. Tur - Eu. Si; Ph] (2989); Ziziphus jujuba Mill [Pol. r; Ph; M, T] (4193). Rosaceae: Agrimonia eupatoria L. [Ir. Tur - Eu. Si; Hem; M] (750); Alchemilla persica Rothm. [Ir. Tur; Helo] (574); Amygdalus lycioides Spach [Ir. Tur; Ph; M, F] (575); Cerasus avium (L.) Moench [Ir. Tur - Eu. Si; Ph; M] (4194); Cerasus incana Spach [Ir. Tur; Ph; M] (576); Cerasus mahaleb Mill. [Ir. Tur - Eu. Si; Ph] (2991); Cerasus microcarpa Boiss. [Ir. Tur; Ph] (577); Cerasus vulgaris Miller [Ir. Tur; Ph; C] (4195); Cotoneaster nummularioides Pojark. [Ir. Tur; Ph; M] (2993); Crataegus azarolus L. subsp. aronia (L.) Riedl. [Ir. Tur; Ph; T] (4196); Cydonia oblonga Mill. [Pol. r; Ph; P, M] (4197); Fragaria vesca L. [Ir. Tur - Eu. Si; Ge-rhiz; M, T, CF,] (4198); Geum kokanicum Regal \& Schmalh [Ir. Tur; Hem; F] (747); Geum urbanum L. [Ir. Tur - Eu. Si; Hem; M, F] (s-n); Malus orientalis Uglitzk. [Ir. Tur; Ph] (2997); Mespilus germanieca L. [Pol. r; Ph; M] (2125); Potentilla recta L. [Ir. Tur Eu. Si; Hem; F] (748); Potentilla supina L. [Ir. Tur - Eu. Si; Hem; F] (s-n); Prunus divaricata Ledeb. [Pol. r; $\mathrm{Ph}$; T] (4199); Rosa canina L. [Ir. Tur - Eu. Si; Ph; M, •] (578); Rosa damascenea Mill. [Ir. Tur; Ph; M, T] (4200); Rosa foetida Herrmann. [Ir. Tur; Ph; F] (579); Rosa orientalis Dupont ex Ser. [Ir. Tur; Ph] (580); Rosa persica Michx. ex Juss. [Ir. Tur - Eu. Si; Ch; F] (2886); Rosa pulverulenta M.Beib. [Ir. Tur - Eu. Si; $\mathrm{Ph}$ ] (749); Rubus caesius L. [Ir. Tur - Eu. Si; Ch; M, T] (2122); Rubus sanctus Schreber. [Ir. Tur - Eu. Si; Ch] (2124); Sanguisorba minor Scop. [Ir. Tur - Eu. Si; Hem; M, F] (581); Sorbus graeca (Spach) Loddinges ex Schauer [Ir. Tur - Eu. Si; Ph] (4201). Rubiaceae: Asperula arvensis L. [Ir. Tur - Eu. Si; Th; F] (4202); Asperula glomerata (M. Bieb) Griseb. [Ir. Tur - Eu. Si; Hem] (582); Asperula taurina L. subsp. caucasica (Pobed.) Ehrend. [Ir. Tur - Eu. Si; Hem; E] (4203); Callipeltis cucullaria (L.) DC. [Ir. Tur - Eu. Si - Sa. Si; Th] (s-n); Cruciata taurica (Pall.) Ehrend. [Ir. Tur; Hem] (583); Galium aparine L. [Ir. Tur; Th; M] (4204); Galium humifusum M.Bieb. [Ir. Tur - Eu. Si; Hem] (4205); Galium mite Boiss. \& Hohen. [Ir. Tur; Hem] (4206); Galium spurium L. [Pol. r; Th] (4207); Galium tricornutum Dandy [Pol. r; Th] (4208); Galium verum L. [Ir. Tur - Eu. Si; Hem; M] (4209). Rutaceae: Haplophyllum perforatuem Kar. \& Kir. [Ir. Tur; Hem; T] (4210). Salicaceae: Salix alba L. [Ir. Tur - Eu. Si; Ph; M] (4211); Salix excelsa J.F.Gmelin [Ir. Tur; Ph; T] (4212); Salix zygostemom Boiss. \& Hohen. [Ir. Tur; Ph] (744). Santalaceae: Thesium kotschyanum Boiss. [Ir. Tur; Hem] (740). Scrophulariaceae: Euphrasia pectinataTen. [Ir. Tur - Eu. Si; Th; M] (584); Leptorhabdos parviflora Benth. [Ir. Tur; Th, •] (s-n); Linaria simplex DC. [Ir. Tur; Th] (4213); Odontites aucheri Boiss. [Ir. Tur; Th] (s-n); Pedicularis pycnantha Boiss. [Ir. Tur; Hem] (4214); Rhynchocorys elephas (L.) Griseb. [Ir. Tur - Eu. Si; Th] (4215); Rhynchocorys maxima Richter. [Ir. Tur - Eu. Si; Th] (4216); Scrophularia azerbaijanica Grau [Ir. Tur - Sa. Si; Hem] (4217); Scrophularia striata Boiss. [Ir. Tur; Hem, -] (585); Scrophularia variegata M.Bieb. [Ir. Tur; Hem, •] (586); Verbascum speciosum Schrad. [Ir. Tur Eu. Si; Hem; M] (s-n); Veronica anagallis - aquatica L. [Pol. r; Hem; M, •] (587); Veronica rechingeri M.A.Fisch. [Ir. Tur - Eu. Si; Th] (2883). Solanaceae: -Datura stramonium L. [Ir. Tur - Eu. Si; Th; M, WP,] 
(588); Hyoscyamus reticulatus L. [Ir. Tur; Hem] (4218); Hyoscyamus senecionis Willd. [Ir. Tur; Hem] (742); Solanum nigrum L. [Ir. Tur - Eu. Si; Th; WP, M,] (589). Tamaricaceae: Myricaria germanica Desv. [Ir. Tur - Eu. Si - Sa. Si; Ph; M] (4219); Tamarix ramosissima Ledeb. [Pol. r; Ph; T] (4220). Thymelaeaceae: Dendrostellera lessertii Tiegh. [Ir. Tur; Ch] (4221); Thymelaea passerina (L.) Cosson \& Germ. [Ir. Tur Eu. Si; Th] (4222). Thyphaceae: Typha minima Funk ex Hoppe [Ir. Tur - Eu. Si; Hem]. Ulmaceae: Celtis cancasica Willd. [Ir. Tur - Eu. Si; Ph] (4223); Ulmus glabra Huds. [Ir. Tur - Eu. Si; Ph] (4224); Ulmus minor Mill. [Ir. Tur - Eu. Si; Ph; M] (4225). Urticaceae: Parietaria judaica L. [Ir. Tur - Eu. Si; Hem; M] (590); Urtica dioica L. [Pol. r; Hem; M, W,P,T] (591). Valerianaceae: Valeriana sisymbriifolia Vahl [Ir. Tur; Hem] (592); Valerianella coronata (L.) DC. [Ir. Tur - Eu. Si; Th] (4226); Valerianella cymbaecarpa C.A.Mey. [Ir. Tur - Eu. Si - Sa. Si; Th] (4227); Vallerianella vesicaria Moench [Ir. Tur - Eu. Si; Th] (4228). Verbenaceae: Verbena officinalis L. [Ir. Tur - Eu. Si; Th; P, M] (4229). Violaceae: Viola arvensis Murray [Ir. Tur - Eu. Si; Th] (4230); Viola modesta Fenzl [Ir. Tur - Eu. Si; Th; T] (4231); Viola odorata L. [Ir. Tur; Hem; M, T] (593). Vitaceae: Vitis vinifera L. [Pol. r; Ph; M]. Zygophyllaceae: Peganum harmala L. [Ir. Tur - Eu. Si; Hem; P, M, F] (2121); Tribulus terrestris L. [Pol. r; Th; WP, F,] (4232); Zygophyllum eurypterum Boiss \& Buhse [Ir. Tur - Eu. Si - Sa. Si; Ph; F] (4233). 